\title{
Spectral Renormalization Group
}

\author{
Jürg Fröhlich * Marcel Griesemer $^{\dagger} \quad$ Israel Michael Sigal $\ddagger \S$
}

November 13, 2008

\begin{abstract}
The operator-theoretic renormalization group (RG) methods are powerful analytic tools to explore spectral properties of field-theoretical models such as quantum electrodynamics (QED) with non-relativistic matter. In this paper these methods are extended and simplified. In a companion paper, our variant of operator-theoretic RG methods is applied to establishing the limiting absorption principle in non-relativistic QED near the ground state energy.
\end{abstract}

\section{Introduction}

This paper is devoted to the nuts and bolts of the spectral (operator-theoretic) renormalization group (RG) method introduced in [7, 8] and developed further in [2, 19]. This method has been used successfully in order to describe the spectral structure of non-relativistic quantum electrodynamics (QED) with confining potentials and of Nelson's model with a 'subcritical' interaction [7, 8, 11, 3, 14, 24] (see [20] for a book exposition and [4, 5, 16], for an alternative multiscale technique). The RG technique developed in this paper is a variant of the one presented in [2], where the smooth Feshbach-Schur map was introduced. It simpler than that of [2] and similar to that of [19].

In this paper we apply the RG technique to prove existence of eigenvaules and describe continuous spectra for operators on Fock spaces appearing in massless quantum field theories for which standard techniques do not work. (The papers [2, 19] deal only

\footnotetext{
*Institute for Theoretical Physics; ETH Zürich; Switzerland; and IHES, Bures-sur-Yvette, France

${ }^{\dagger}$ Department of Mathematics, University of Stuttgart, D-70569 Stuttgart, Germany

†Department of Mathematics, University of Toronto; Toronto; Canada

$\S$ Supported by NSERC Grant No. NA7901
} 
with eigenvalues.) The results obtained here are used in subsequent papers to prove existence of the ground state and resonances for non-relativistic QED without the confinement assumption ([24], see also [4] ) and to prove local decay near the ground state energy ([17], see also [16]).

The class of Hamiltonians and the problems we consider here originate in non-relativistic QED. This theory deals with the interactions of non-relativistic matter with the quantized electro-magnetic field. (See [12, 13, 20, 25] for background.)

The dynamics of non-relativistic matter is generated by the Schrödinger operator

$$
H_{p}:=-\sum_{j=1}^{n} \frac{1}{2 m_{j}} \Delta_{x_{j}}+V(x),
$$

where $\Delta_{x_{j}}$ is the Laplacian in the variable $x_{j}, x=\left(x_{1}, \ldots, x_{n}\right)$, and $V(x)$ is the potential energy of the particle system. This operator acts on the Hilbert space $\mathcal{H}_{p}$, which is either $L^{2}\left(\mathbb{R}^{3 n}\right)$ or a subspace of this space determined by a symmetry group of the particle system. We assume that $V(x)$ is real and s.t. the operator $H_{p}$ is self-adjoint.

The quantized electromagnetic field is described by the quantized vector potential

$$
A(y)=\int\left(e^{i k y} a(k)+e^{-i k y} a^{*}(k)\right) \chi(k) \frac{d^{3} k}{\sqrt{|k|}}
$$

in the Coulomb gauge $(\operatorname{div} A(x)=0)$. Here $\chi$ is an ultraviolet cut-off: $\chi(k)=\frac{1}{(2 \pi)^{3} \sqrt{2}}$ in a neighborhood of $k=0$, and $\chi$ vanishes rapidly at infinity. The dynamics of the quantized electromagnetic field is given by the quantum Hamiltonian

$$
H_{f}=\int d^{3} k \omega(k) a^{*}(k) a(k) .
$$

The operators $A(y)$ and $H_{p}$ act on the Fock space $\mathcal{H}_{f} \equiv \mathcal{F}$. Above, $\omega(k)=|k|$ is the dispersion law connecting the energy, $\omega(k)$, of the field quantum with its wave vector $k$, and $a^{*}(k)$ and $a(k)$ denote the creation and annihilation operators on $\mathcal{F}$. The latter are operator-valued generalized, transverse vector fields:

$$
a^{\#}(k):=\sum_{\lambda \in\{0,1\}} e_{\lambda}(k) a_{\lambda}^{\#}(k),
$$

where $e_{\lambda}(k)$ are polarization vectors, i.e. orthonormal vectors in $\mathbb{R}^{3}$ satisfying $k \cdot e_{\lambda}(k)=$ 0 , and $a_{\lambda}^{\#}(k)$ are scalar creation and annihilation operators satisfying canonical commutation relations. The right side of $(\overline{I .3})$ can be understood as a weak integral. See the 
Supplement for a brief review of definitions of the Fock space, the creation and annihilation operators and the operator $H_{f}$.

The Hamiltonian of the total system, matter and radiation field, is given by

$$
H_{g}=\sum_{j=1}^{n} \frac{1}{2 m_{j}}\left(-i \nabla_{x_{j}}+g A\left(x_{j}\right)\right)^{2}+V(x)+H_{f}
$$

acting on the Hilbert space $\mathcal{H}:=\mathcal{H}_{p} \otimes \mathcal{H}_{f}$. Here the coupling constant $g$ is related to the fine-structure constant $\alpha=\frac{e^{2}}{4 \pi \hbar c} \approx \frac{1}{137}$. (See [9, 16, 24] for a discussion of the definition of $H_{g}$ and units involved.) This model describes emission and absorption of radiation by systems of matter, such as atoms and molecules, as well as other processes of interaction of quantized radiation with matter. It has been extensively studied in the last decade; see references in [24, 25] for references to earlier contributions.

For a large class of potentials $V(x)$, including Coulomb potentials, and for an ultraviolet cut-off in $A(x)$, the operator $H_{g}$ is self-adjoint.

The key problem of non-relativistic QED is to establish spectral and resonance structure of $H_{g}$ and, in particular, to prove existence (and uniqueness) of the ground state and of resonances of $H_{g}$ corresponding to excited states of the atomic Hamiltonian.

One verifies that $H_{f}$ defines a positive, self-adjoint operator on $\mathcal{F}$ with purely absolutely continuous spectrum, except for a simple eigenvalue 0 corresponding to the vacuum eigenvector $\Omega$ (see Supplement). Thus, for $g=0$, the low-energy spectrum of the Hamiltonian $H_{0}$ of the decoupled system consists of branches $\left[\epsilon_{i}^{(p)}, \infty\right)$ of absolutely continuous spectrum, where $\epsilon_{i}^{(p)}$ are the isolated eigenvalues of the particle Hamiltonian $H_{p}$, and of the eigenvalues $\epsilon_{i}^{(p)}$ sitting at the 'thresholds' of the continuous spectrum. The absence of gaps between the eigenvalues and thresholds is a consequence of the fact that the photons are massless. This leads to hard and subtle problems in perturbation theory, known collectively as the infrared problem.

The first step in tackling the problem of ground states and resonances in the framework of the RG approach is to perform a certain canonical transformation and then apply to the resulting Hamiltonian a specially designed RG map in order to project out the particleand high-photon-energy degrees of freedom ([24] (cf. [7]). As a result, one arrives at a Hamiltonian on Fock space of the form $H:=T+W$, where $T:=w_{0,0}\left[H_{f}\right]$, with $w_{0,0}:[0, \infty) \rightarrow \mathbb{C}$ and continuous $\left(w_{0,0}\left[H_{f}\right]\right.$ is defined by the operator calculus $)$, and

$$
\begin{aligned}
W:= & \sum_{m+n \geq 1} \chi_{1} \int_{B_{1}^{m+n}} \prod_{1}^{m+n}\left(\frac{d k_{j}}{\left|k_{j}\right|^{1 / 2}}\right) \prod_{1}^{m} a^{*}\left(k_{j}\right) \\
& \times w_{m, n}\left[H_{f} ; k_{1}, \ldots, k_{m+n}\right] \prod_{m+1}^{m+n} a\left(k_{j}\right) \chi_{1}
\end{aligned}
$$


Here $w_{m, n}: I \times B_{1}^{m+n} \rightarrow \mathbb{C}, m+n>0, B_{1}^{r}$ denotes the Cartesian product of $r$ unit balls in $\mathbb{R}^{3}, I:=[0,1]$ and $\chi_{1}:=\chi_{1}\left(H_{f}\right)$ with $\chi_{1}(r)$ a smooth cut-off function s.t. $\chi_{1}=1$ for $r \leq 9 / 10,=0$ for $r \geq 1$ and $0 \leq \chi_{1}(r) \leq 1$. See Section $\amalg$ for more details concerning notation. Operators on Fock space of the form above will be said to be in generalized normal (or Wick) form.

Note that, in order to be able to apply our theory to the analysis of resonances of $H_{g}$, the operators $H=T+W$, introduced above, are allowed to be non-self-adjoint.

Our goal in this paper is to describe the spectrum of the operator $H$ near 0 . We assume that the function $w_{0,0}(r)$, defining the operator $T:=w_{0,0}\left[H_{f}\right]$, satisfies

$$
w_{0,0}(0)=0, \sup _{r \in[0, \infty)}\left|w_{0,0}^{\prime}(r)-1\right| \leq \beta_{0}
$$

We consider the operator $W\left(\right.$ see $(\underline{\mathrm{I} .5})$ ) as a perturbation of the operator $T:=w_{0,0}\left[H_{f}\right]$, whose spectrum is explicitly known. It consists of the essential spectrum $w_{0,0}\left(\overline{\mathbb{R}^{+}}\right)$and an eigenvalue 0 at its tip with the eigenvector $\Omega$. We propose to determine the effect of the perturbation $W$ on the spectrum of $T$ near 0 and, in particular, to determine the fate of the eigenvalue 0 of $T$. If the operator $H$ has an eigenvalue near 0 , we call it the ground state energy of $H$.

We denote by $\mathcal{D}_{s}$ the set of operators of the form $H=T+W$, where $T$ and $W$ are described above, such that (I.6) holds and

$$
\left\|\underline{w}_{1}\right\|_{\mu, s, \xi} \leq \gamma_{0}
$$

where $\underline{w}_{1}:=\left(w_{m, n}\right)_{m+n \geq 1}$, and $\left\|\underline{w}_{1}\right\|_{\mu, s, \xi}$ is a norm defined in Section III. We define a subset $S$ of the complex plane by

$$
S:=\left\{w \in \mathbb{C}|\operatorname{Re} w \geq 0,| \operatorname{Im} w \mid \leq \frac{1}{3} \operatorname{Re} w\right\} .
$$

Recall that a complex function $f$ on an open set $\mathcal{D}$ in a complex Banach space $\mathcal{B}$ is said to be analytic if $\forall H \in \mathcal{D}$ and $\forall \xi \in \mathcal{B}, f(H+\tau \xi)$ is analytic in the complex variable $\tau$ for $|\tau|$ sufficiently small (or equivalently, $f$ is Gâteaux-differentiable, see [10]; a stronger notion of analyticity, requiring in addition that $f$ is locally bounded, is used in [21]). In the next theorem $\mathcal{B}$ is the space of $H_{f}$-bounded operators on $\mathcal{F}$ (i.e. the space of closed operators $A$ with $A\left(H_{f}+1\right)^{-1}$ bounded). We are now prepared to state the main result of this paper.

Theorem I.1. Assume that $\beta_{0}$ and $\gamma_{0}$ are sufficiently small. Then there is an analytic map $e: \mathcal{D}_{s} \rightarrow \mathbb{C}$ such that $e(H) \in \mathbb{R}$, for $H=H^{*}$, and for $H \in \mathcal{D}_{s}$ the number $e(H)$ is a simple eigenvalue of the operator $H$ and $\sigma(H) \subset e(H)+S$. 
Note that our approach also provides an effective way to compute the eigenvalue $e(H)$ and the corresponding eigenvector.

Theorem I.1 is used in [24, 17]. Besides, our main technical result, Theorem V.1 formulated in Section $\nabla$, furnishes a key technical step in an RG proof of local decay, see [17].

Combining results of this paper with those of [1] one obtains estimates on the resolvent of $H$ near the eigenvalue $e(H)$ : For each $\Psi$ and $\Phi$ from a dense set of vectors, the matrix element $\left\langle\Psi,(H-z)^{-1} \Phi\right\rangle$ near the eigenvalue $e \equiv e(H)$ of $H$ is of the form

$$
\left\langle\Psi,(H-z)^{-1} \Phi\right\rangle=(e-z)^{-1} p(\Psi, \Phi)+r(z, \Psi, \Phi),
$$

where $p$ and $r(z)$ are sesquilinear forms in $\Psi$ and $\Phi$ with $r(z)$ analytic in $z \in Q:=$ $\mathbb{C} \backslash(e(H)+S)$ and bounded on the intersection of a neighbourhood of $e$ with $Q$ as

$$
|r(z, \Psi, \Phi)| \leq C_{\Psi, \Phi}|e-z|^{-\gamma} \text { for some } \gamma<1
$$

Such estimates are needed in an analysis of the long time dynamics of resonances in QED; see [1]. This will be described in more detail elsewhere.

Next, we explain the main ideas of the spectral renormalization group method. Our goal is to describe the spectral structure near 0 of an operator $H$ from the set $\mathcal{D}_{s}$ introduced above. Denote by $D(0, \alpha)$ the disc in $\mathbb{C}$ centered at 0 and of radius $\alpha$. For $\alpha_{0}$ sufficiently small, we construct a renormalization transformation, $\mathcal{R}_{\rho}$, defined on $\mathcal{D}:=D\left(0, \alpha_{0}\right) \mathbf{1}+$ $\mathcal{D}_{s}$, with the following properties:

- $\mathcal{R}_{\rho}$ is 'isospectral' and 'preserves' the limiting absorption principle;

- $\mathcal{R}_{\rho}$ removes the photon degrees of freedom related to energies $\geq \rho$.

We then consider the discrete semi-flow, $\mathcal{R}_{\rho}^{n}, n \geq 1$, generated by the renormalization transformation, $\mathcal{R}_{\rho}$ (called renormalization group) and relate the dynamics of this flow to spectral properties of individual Hamiltonians in $\mathcal{D}_{s}$. We show that the flow, $\mathcal{R}_{\rho}^{n}$, has the fixed-point manifold $\mathcal{M}_{f p}:=\mathbb{C} H_{f}$, an unstable manifold $\mathcal{M}_{u}:=\mathbb{C} 1$, and a (complex) co-dimension 1 stable manifold $\mathcal{M}_{s}$ for $\mathcal{M}_{f p}$ foliated by (complex) co-dimension 2 stable manifolds for each fixed point. We show that $H-\lambda$ is in the domain of $\mathcal{R}_{\rho}^{n}$, provided the parameter $\lambda$ is adjusted appropriately, so that $H-\lambda$ is, roughly, in a $\rho^{n}$-neighborhood of the stable manifold $\mathcal{M}_{s}$. 


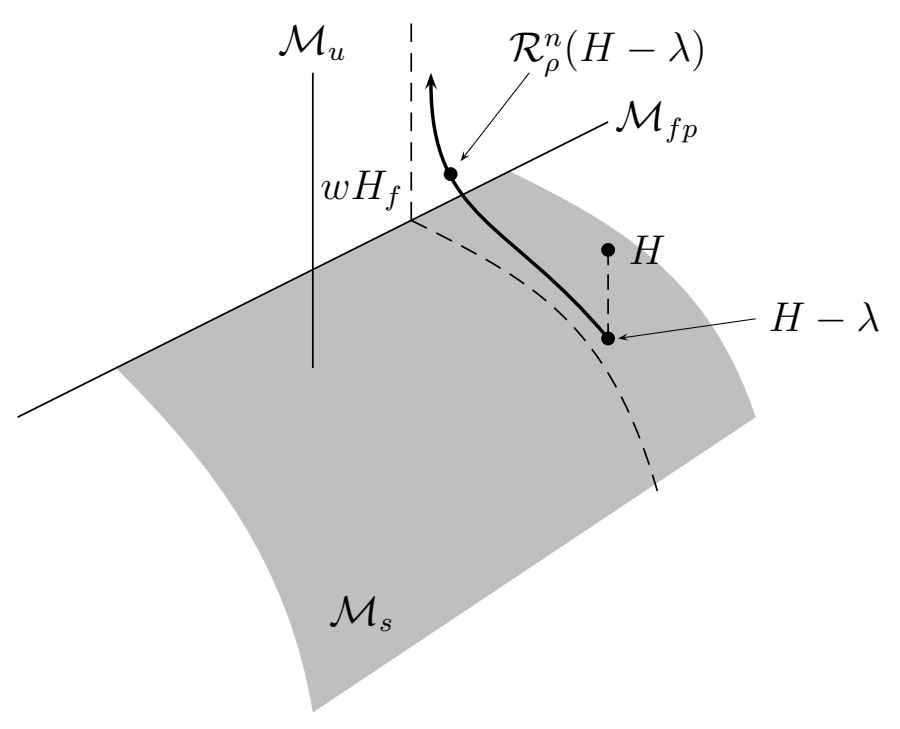

Stable and unstable manifolds.

Thus, for $n$ sufficiently large, the operators $H_{\lambda}^{(n)}:=\mathcal{R}_{\rho}^{n}(H-\lambda)$ are close to the operator $w H_{f}$, for some $w \in \mathbb{C}$ with Re $w>0$, and their spectra can be easily analyzed. Since the renormalization map is 'isospectral', we can pass this spectral information to the operator $H_{\lambda}^{(n-1)}$, and so forth, until we obtain the desired spectral information for the initial operator $H$.

Our paper is organized as follows. In Section $\Pi$ we describe the Feshbach-Schur map, which is the main ingredient of the renormalization map introduced in Section [V] In Section [II we define the Banach spaces on which the renormalization map acts. The renormalization group approach is presented in Section $\nabla$ where the main technical results implying Theorem I.1 are proven. In Appendix I we present the proof of a key technical result describing properties of the renormalization map. This proof is close to the proof of a similar result in [2] and is presented here for the reader's convenience. In Appendix II we present a result on the construction of eigenvalues and eigenvectors, similar to a corresponding result of [2]. Finally, in a Supplement, we collect some relevant facts on Fock space and creation and annihilation operators. 


\section{The Smooth Feshbach-Schur Map}

In this section, we review the method of isospectral decimation maps acting on operators, introduced in [7, 8] and refined in [2]. At the origin of this method is the isospectral smooth Feshbach-Schur map 11 acting on a set of closed operators and mapping a given operator to one acting on a subspace of the original Hilbert space.

Let $\chi, \bar{\chi}$ be a partition of unity on a separable Hilbert space $\mathcal{H}$, i.e. $\chi$ and $\bar{\chi}$ are positive operators on $\mathcal{H}$ whose norms are bounded by one, $0 \leq \chi, \bar{\chi} \leq 1$, and $\chi^{2}+\bar{\chi}^{2}=1$. We assume that $\chi$ and $\bar{\chi}$ are nonzero. Let $\tau$ be a (linear) projection acting on closed operators on $\mathcal{H}$ with the property that operators in its image commute with $\chi$ and $\bar{\chi}$. We also assume that $\tau(\mathbf{1})=\mathbf{1}$. Let $\bar{\tau}:=\mathbf{1}-\tau$ and define

$$
H_{\tau, \chi^{\#}}:=\tau(H)+\chi^{\#} \bar{\tau}(H) \chi^{\#} .
$$

where $\chi^{\#}$ stands for either $\chi$ or $\bar{\chi}$.

Given $\chi$ and $\tau$ as above, we denote by $D_{\tau, \chi}$ the space of closed operators, $H$, on $\mathcal{H}$ which belong to the domain of $\tau$ and satisfy the following three conditions:

(i) $\tau$ and $\chi$ (and therefore also $\bar{\tau}$ and $\bar{\chi}$ ) leave the domain $D(H)$ of $H$ invariant:

$$
D(\tau(H))=D(H) \text { and } \chi D(H) \subset D(H),
$$

(ii)

$$
H_{\tau, \bar{\chi}} \text { is (bounded) invertible on } \operatorname{Ran} \bar{\chi}
$$

and

(iii)

$$
\bar{\tau}(H) \chi \text { and } \chi \bar{\tau}(H) \text { extend to bounded operators on } \mathcal{H} \text {. }
$$

(For more general conditions see [2, 18].)

The smooth Feshbach-Schur map (SFM) maps operators on $\mathcal{H}$ belonging to $D_{\tau, \chi}$ to operators on $\mathcal{H}$ by $H \mapsto F_{\tau, \chi}(H)$, where

$$
F_{\tau, \chi}(H):=H_{0}+\chi W \chi-\chi W \bar{\chi} H_{\tau, \bar{\chi}}^{-1} \bar{\chi} W \chi .
$$

Here $H_{0}:=\tau(H)$ and $W:=\bar{\tau}(H)$. Note that $H_{0}$ and $W$ are closed operators on $\mathcal{H}$ with coinciding domains, $D\left(H_{0}\right)=D(W)=D(H)$, and $H=H_{0}+W$. We remark that the domains of $\chi W \chi, \bar{\chi} W \bar{\chi}, H_{\tau, \chi}$, and $H_{\tau, \bar{\chi}}$ all contain $D(H)$.

Remarks

\footnotetext{
${ }^{1}$ In [7, 8, 2] this map is called the Feshbach map. As was pointed out to us by F. Klopp and B. Simon, the invertibility procedure at the heart of this map was introduced by I. Schur in 1917; it appeared implicitly in an independent work of H. Feshbach on the theory of nuclear reactions, in 1958, where the problem of perturbations of operator eigenvalues was considered. See [18] for further extensions and historical remarks.
} 
- The definition of the smooth Feshbach map given above differs somewhat from the one given in [2]. In [2], the map $F_{\tau, \chi}(H)$ is denoted by $F_{\chi}(H, \tau(H))$, and the pair of operators $(H, T)$ are referred to as a Feshbach pair.

- The usual Feshbach-Schur map is obtained as a special case of the smooth FeshbachSchur map by choosing $\chi=$ projection, and, usually, $\tau=0$.

- Typically the operator $\chi$ is taken to be of the form $\chi:=\chi(A)$ for some self-adjoint operator $A$ on $\mathcal{H}$. For the Feshbach map, $\chi$ has to be a projection and therefore we would have to take $\chi:=\chi(A)$ to be a characteristic function of the operator $A$, while in the smooth Feshbach-Schur map we are allowed to take $\chi:=\chi(A)$ to be a smooth approximation of the characteristic function of an interval in $\mathbb{R}$. This explains the adjective 'smooth' in the definition.

- In [2] a semi-group property of $F_{\tau, \chi}(H)$ is exhibited.

Next, we introduce some maps appearing in various identities involving the FeshbachSchur map:

$$
\begin{aligned}
& Q_{\tau, \chi}(H):=\chi-\bar{\chi} H_{\tau, \bar{\chi}}^{-1} \bar{\chi} W \chi \\
& Q_{\tau, \chi}^{\#}(H):=\chi-\chi W \bar{\chi} H_{\tau, \bar{\chi}}^{-1} \bar{\chi} .
\end{aligned}
$$

Note that $Q_{\tau, \chi}(H) \in \mathcal{B}(\operatorname{Ran} \chi, \mathcal{H})$ and $Q_{\tau, \chi}^{\#}(H) \in \mathcal{B}(\mathcal{H}, \operatorname{Ran} \chi)$.

The smooth Feshbach-Schur map of $H$ is isospectral to $H$ in the sense of the following theorem.

Theorem II.1. Let $\chi$ and $\tau$ be as above, and assume that $H \in D_{\tau, \chi}$ so that $F_{\tau, \chi}(H)$ is well defined. Then

(i) $0 \in \rho(H) \Leftrightarrow 0 \in \rho\left(F_{\tau, \chi}(H)\right)$, i.e. $H$ is bounded invertible on $\mathcal{H}$ if and only if $F_{\tau, \chi}(H)$ is bounded invertible on $\operatorname{Ran} \chi$.

(ii) If $\psi \in \mathcal{H} \backslash\{0\}$ solves $H \psi=0$ then $\varphi:=\chi \psi \in \operatorname{Ran} \chi \backslash\{0\}$ solves $F_{\tau, \chi}(H) \varphi=0$.

(iii) If $\varphi \in \operatorname{Ran} \chi \backslash\{0\}$ solves $F_{\tau, \chi}(H) \varphi=0$ then $\psi:=Q_{\tau, \chi}(H) \varphi \in \mathcal{H} \backslash\{0\}$ solves $H \psi=0$.

(iv) The multiplicity of the spectral value $\{0\}$ is conserved under the Feshbach-Schur in the sense that $\operatorname{dim} \operatorname{Ker} H=\operatorname{dim} \operatorname{Ker} F_{\tau, \chi}(H)$. 
(v) If one of the inverses, $H^{-1}$ or $F_{\tau, \chi}(H)^{-1}$, exists then so does the other, and these inverses are related by

$$
H^{-1}=Q_{\tau, \chi}(H) F_{\tau, \chi}(H)^{-1} Q_{\tau, \chi}(H)^{\#}+\bar{\chi} H_{\tau, \bar{\chi}}^{-1} \bar{\chi} .
$$

Moreover if $\tau(H)$ is invertible, then

$$
F_{\tau, \chi}(H)^{-1}=\chi H^{-1} \chi+\bar{\chi} \tau(H)^{-1} \bar{\chi} .
$$

This theorem is proven in [2]; see [18] for further extensions.

In comparison with the original use of the Feshbach projection method as a tool in the analytic perturbation theory of eigenvalues, the smooth Feshbach-Schur map has two new features:

- Flexibility in the choice of the projection; in particular, 'dressing' the eigenspace corresponding to some eigenvalue with vectors from the continuous spectrum subspace, and relaxing the projection property altogether;

- Viewing the Feshbach-Schur procedure as a map on a space of operators, rather then a tool in the analysis of a fixed operator. Our operator theoretic renormalization group is based on an iterative composition of Feshbach-Schur maps, decimating the degrees of freedom of the system under investigation.

\section{A Banach Space of Hamiltonians}

We construct a Banach space of Hamiltonians on which our renormalization transformation will be defined. In order not to complicate matters unnecessarily, we will think of the creation and annihilation operators used below as scalar operators neglecting helicity of photons. We explain at the end of the Supplement how to reinterpret our expressions for the photon creation and annihilation operators.

Recall that $B_{1}^{r}$ denotes the Cartesian product of $r$ unit balls in $\mathbb{R}^{3}, I:=[0,1]$ and $m, n \geq 0$. Given functions $w_{0,0}:[0, \infty) \rightarrow \mathbb{C}$ and $w_{m, n}: I \times B^{m+n} \rightarrow \mathbb{C}, m+n>0$, we consider monomials, $W_{m, n} \equiv W_{m, n}\left[w_{m, n}\right]$, in the creation and annihilation operators defined as follows: $W_{0,0}\left[w_{0,0}\right]:=w_{0,0}\left[H_{f}\right]$ (defined by the functional calculus), and

$$
\begin{aligned}
& W_{m, n}\left[w_{m, n}\right]:= \\
& \int_{B_{1}^{m+n}} \frac{d k_{(m, n)}}{\left|k_{(m, n)}\right|^{1 / 2}} a^{*}\left(k_{(m)}\right) w_{m, n}\left[H_{f} ; k_{(m, n)}\right] a\left(\tilde{k}_{(n)}\right),
\end{aligned}
$$


for $m+n>0$. Here we are using the notation

$$
\begin{gathered}
k_{(m)}:=\left(k_{1}, \ldots, k_{m}\right) \in \mathbb{R}^{3 m}, \quad a^{*}\left(k_{(m)}\right):=\prod_{i=1}^{m} a^{*}\left(k_{i}\right), \\
k_{(m, n)}:=\left(k_{(m)}, \tilde{k}_{(n)}\right), \quad d k_{(m, n)}:=\prod_{i=1}^{m} d^{3} k_{i} \prod_{i=1}^{n} d^{3} \tilde{k}_{i}, \\
\left|k_{(m, n)}\right|:=\left|k_{(m)}\right| \cdot\left|\tilde{k}_{(n)}\right|, \quad\left|k_{(m)}\right|:=\left|k_{1}\right| \cdots\left|k_{m}\right| .
\end{gathered}
$$

The notation $W_{m, n}\left[w_{m, n}\right]$ stresses the dependence of $W_{m, n}$ on $w_{m, n}$. Note that $W_{0,0}\left[w_{0,0}\right]$ $:=w_{0,0}\left[H_{f}\right]$. We also denote $T \equiv W_{0,0}\left[w_{0,0}\right]$.

We assume that, for every $m$ and $n$ with $m+n>0$, the function $w_{m, n}\left[r ;, k_{(m, n)}\right]$ is measurable in $k_{(m, n)} \in B_{1}^{m+n}$ and $s$ times continuously differentiable in $r \in I$, for some $s \geq 1$, and for almost every $k_{(m, n)} \in B_{1}^{m+n}$. As a function of $k_{(m, n)}$, it is totally symmetric w. r. t. the variables $k_{(m)}=\left(k_{1}, \ldots, k_{m}\right)$ and $\tilde{k}_{(n)}=\left(\tilde{k}_{1}, \ldots, \tilde{k}_{n}\right)$ and obeys the norm bound

$$
\left\|w_{m, n}\right\|_{\mu, s}:=\sum_{n=0}^{s}\left\|\partial_{r}^{n} w_{m, n}\right\|_{\mu}<\infty
$$

where

$$
\left\|w_{m, n}\right\|_{\mu}:=\left.\max _{j} \sup _{r \in I, k_{(m, n)} \in B_{1}^{m+n}}|| k_{j}\right|^{-\mu} w_{m, n}\left[r ; k_{(m, n)}\right] \mid
$$

for some $\mu \geq 0$. Here and in what follows, $k_{j}$ is one of the $3-$ vectors in the variable $k_{(m, n)}$. Recall that $\left|k_{(m, n)}\right|^{-1 / 2}$ is absorbed in the integration measure in the definition of $W_{m, n}$. For $m+n=0$ the variable $r$ ranges over $[0, \infty)$, and we assume that the following norm is finite:

$$
\left\|w_{0,0}\right\|_{\mu, s}:=\left|w_{0,0}(0)\right|+\sum_{1 \leq n \leq s} \sup _{r \in[0, \infty)}\left|\partial_{r}^{n} w_{0,0}(r)\right| .
$$

(This norm is independent of $\mu$, but we keep this index for notational convenience.) The Banach space of functions $w_{m, n}$ of this type is denoted by $\mathcal{W}_{m, n}^{\mu, s}$.

We fix three numbers $\mu, 0<\xi<1$ and $s \geq 0$ and define the Banach space

$$
\mathcal{W}^{\mu, s} \equiv \mathcal{W}_{\xi}^{\mu, s}:=\bigoplus_{m+n \geq 0} \mathcal{W}_{m, n}^{\mu, s},
$$

with the norm

$$
\|\underline{w}\|_{\mu, s, \xi}:=\sum_{m+n \geq 0} \xi^{-(m+n)}\left\|w_{m, n}\right\|_{\mu, s}<\infty .
$$

Clearly, $\mathcal{W}_{\xi^{\prime}}^{\mu^{\prime}, s^{\prime}} \subset \mathcal{W}_{\xi}^{\mu, s}$ if $\mu^{\prime} \geq \mu, s^{\prime} \geq s$ and $\xi^{\prime} \leq \xi$.

Let $\chi_{1}(r) \equiv \chi_{r \leq 1}$ be a smooth cut-off function s.t. $\chi_{1}=1$ for $r \leq 9 / 10,=0$ for $r \geq 1$ and $0 \leq \chi_{1}(r) \leq 1$ and $\sup \left|\partial_{r}^{n} \chi_{1}(r)\right| \leq 30 \forall r$ and for $n=1,2$. We define 
$\chi_{\rho}(r) \equiv \chi_{r \leq \rho}:=\chi_{1}(r / \rho) \equiv \chi_{r / \rho \leq 1}$ and $\chi_{\rho} \equiv \chi_{H_{f} \leq \rho}$. The following basic bound, proven in [2], links the norm defined in (III.6) to the operator norm on $\mathcal{B}[\mathcal{F}]$.

Theorem III.1. Fix $m, n \in \mathbb{N}_{0}$ such that $m+n \geq 1$. Suppose that $w_{m, n} \in \mathcal{W}_{m, n}^{\mu, s}$, and let $W_{m, n} \equiv W_{m, n}\left[w_{m, n}\right]$ be as defined in (III.1). Then for all $\lambda>0$

$$
\left\|\left(H_{f}+\lambda\right)^{-m / 2} W_{m, n}\left(H_{f}+\lambda\right)^{-n / 2}\right\| \leq\left\|w_{m, n}\right\|_{0}
$$

and therefore

$$
\left\|\chi_{\rho} W_{m, n} \chi_{\rho}\right\| \leq \frac{\rho^{(m+n)(1+\mu)}}{\sqrt{m ! n !}}\left\|w_{m, n}\right\|_{0},
$$

where $\|\cdot\|$ denotes the operator norm on $\mathcal{B}[\mathcal{F}]$.

Theorem III.1 says that the finiteness of $\left\|w_{m, n}\right\|_{0}$ insures that $\chi_{1} W_{m, n} \chi_{1}$ defines a bounded operator on $\mathcal{B}[\mathcal{F}]$.

With a sequence $\underline{w}:=\left(w_{m, n}\right)_{m+n \geq 0}$ in $\mathcal{W}^{\mu, s}$ we associate an operator by setting

$$
H(\underline{w}):=W_{0,0}[\underline{w}]+\sum_{m+n \geq 1} \chi_{1} W_{m, n}[\underline{w}] \chi_{1}
$$

where we write $W_{m, n}[\underline{w}]:=W_{m, n}\left[w_{m, n}\right]$. These operators are said to be in generalized normal (or Wick) form and are called generalized Wick-ordered operators. Theorem III.1 shows that the series in (III.12) converges in the operator norm and obeys the estimate

$$
\left\|H(\underline{w})-W_{0,0}(\underline{w})\right\| \leq \xi\left\|\underline{w}_{1}\right\|_{\mu, 0, \xi}
$$

for arbitrary $\underline{w}=\left(w_{m, n}\right)_{m+n \geq 0} \in \mathcal{W}^{\mu, 0}$ and any $\mu>-1 / 2$. Here $\underline{w}_{1}=\left(w_{m, n}\right)_{m+n \geq 1}$. Hence we have the linear map

$$
H: \underline{w} \rightarrow H(\underline{w})
$$

from $\mathcal{W}^{\mu, 0}$ into the set of closed operators on Fock space $\mathcal{F}$. The following result is proven in [2].

Theorem III.2. For any $\mu \geq 0$ and $0<\xi<1$, the map $H: \underline{w} \rightarrow H(\underline{w})$, given in (III.12), is injective.

Next, we decompose the Banach space $\mathcal{W}^{\mu, s}$ into components having, as we will establish below, distinct scaling properties. We define the Banach spaces

$$
\mathcal{T}:=\left\{f \in \mathcal{W}_{0,0}^{\mu, s} \mid f(0)=0\right\}
$$


and

$$
\mathcal{W}_{1}^{\mu, s}:=\bigoplus_{m+n \geq 1} \mathcal{W}_{m, n}^{\mu, s}
$$

to consist of all sequences $\underline{w}_{1}:=\left(w_{m, n}\right)_{m+n \geq 1}$ obeying

$$
\left\|\underline{w}_{1}\right\|_{\mu, s, \xi}:=\sum_{m+n \geq 1} \xi^{-(m+n)}\left\|w_{m, n}\right\|_{\mu, s}<\infty .
$$

We observe that there is a natural bijection

$$
\mathcal{W}_{0,0}^{\mu, s} \rightarrow \mathbb{C} \oplus \mathcal{T}, w_{0,0} \mapsto w_{0,0}[0] \oplus\left(w_{0,0}-w_{0,0}[0]\right)
$$

We shall henceforth not distinguish between $\mathcal{W}_{0,0}^{\mu, s}$ and $\mathbb{C} \oplus \mathcal{T}$. We rewrite our Banach $\mathcal{W}^{\mu, s}$ space as

$$
\mathcal{W}^{\mu, s}=\mathbb{C} \oplus \mathcal{T} \oplus \mathcal{W}_{1}^{\mu, s}
$$

We define the spaces $\mathcal{W}_{o p}^{\mu, s}:=H\left(\mathcal{W}^{\mu, s}\right), \mathcal{W}_{1, o p}^{\mu, s}:=H\left(\mathcal{W}_{1}^{\mu, s}\right)$ and $\mathcal{W}_{m n, o p}^{\mu, s}:=H\left(\mathcal{W}_{m n}^{\mu, s}\right)$. Sometimes we display the parameter $\xi$, as in $\mathcal{W}_{o p, \xi}^{\mu, s}:=H\left(\mathcal{W}_{\xi}^{\mu, s}\right)$. Theorem $\amalg$ II.2 implies that $H\left(\mathcal{W}^{\mu, s}\right)$ is a Banach space with norm $\|H(\underline{w})\|_{\mu, s, \xi}:=\|\underline{w}\|_{\mu, s, \xi}$.

Corresponding to (III.18), operators in $\mathcal{W}_{o p}^{\mu, s}$ can be represented as

$$
H(\underline{w})=E \mathbf{1}+T+W
$$

where $E \in \mathbb{C}$ is a complex number, $T=T\left[H_{f}\right]$, with $T[.] \in \mathcal{T}$, and $W \in \mathcal{W}_{1}^{\mu, s}$. Indeed, let

$$
E:=w_{0,0}[0], T:=w_{0,0}\left[H_{f}\right]-w_{0,0}[0] \text { and } W:=\sum_{m+n \geq 1} \chi_{1} W_{m, n}[\underline{w}] \chi_{1}
$$

Then the equation (III.19) holds.

Remark III.3. In this paper we need only $s=1$. We introduce the more general spaces for the sake of future references. Indeed, in our proof the limiting absorption principle $(L A P)$ in [17] we need $s=2$. More precisely, we have to use more sophisticated Banach spaces where the operator $\partial_{r}^{n}$ in (III.5), is replaced by the operator $\partial_{r}^{n}\left(k \partial_{k}\right)^{q}$ to (VI.16). Here $q:=\left(q_{1}, \ldots, q_{M+N}\right),\left(k \partial_{k}\right)^{q}:=\prod_{1}^{M+N}\left(k_{j} \cdot \nabla_{k_{j}}\right)^{q_{j}}$, with $k_{m+j}:=\tilde{k}_{j}$, and the indices $n$ and $q$ satisfy $0 \leq n+|q| \leq s$ with $s=2$. 


\section{The Renormalization Transformation $\mathcal{R}_{\rho}$}

In this section we introduce an operator-theoretic renormalization transformation based on the smooth Feshbach-Schur map, which is closely related to the one introduced in [2] and [7, 8]. We fix the index $\mu$ in our Banach spaces at some positive value, $\mu>0$.

The renormalization transformation is homothetic to an isospectral map defined on a polydisc in a suitable Banach space of Hamiltonians. It has a certain contraction property insuring that (upon appropriate tuning of the spectral parameter) the image of any Hamiltonian in the polydisc under a large number of iterations of the renormalization transformation approaches a fixed-point Hamiltonian, $w H_{f}$, whose spectral analysis is particularly simple. Thanks to the isospectrality of the renormalization map, certain properties of the spectrum of the initial Hamiltonian can be derived from the corresponding properties of the limiting Hamiltonian.

The renormalization map is defined below as a composition of a decimation map, $F_{\rho}$, and two rescaling maps, $S_{\rho}$ and $A_{\rho}$. Here $\rho$ is a positive parameter - the photon energy scale - which will be chosen later.

The decimation of degrees of freedom is accomplished by the smooth Feshbach map, $F_{\tau, \chi}$ with the operators $\tau$ and $\chi$ chosen as

$$
\tau(H)=W_{00}:=w_{00}\left(H_{f}\right) \text { and } \chi=\chi_{\rho} \equiv \chi_{H_{f} \leq \rho},
$$

where $H=H(\underline{w})$ is given in Eqn (III.12). With $\tau$ and $\chi$ identified in this way we will use the notation

$$
F_{\rho} \equiv F_{\tau, \chi_{\rho}}
$$

The decimation map acts on the Banach space $\mathcal{W}_{o p}^{s}$

Let $\bar{\chi}_{\rho}$ be defined so that $\chi_{\rho} \equiv \chi_{H_{f} \leq \rho}$ and $\bar{\chi}_{\rho} \equiv \chi_{H_{f} \geq \rho}$ form a smooth partition of unity, $\chi_{\rho}^{2}+\bar{\chi}_{\rho}^{2}=1$. The lemma below shows that the domain of this map contains the following polydisc in $\mathcal{W}_{o p}^{\mu, s}$ :

$$
\begin{aligned}
& \mathcal{D}^{\mu, s}(\alpha, \beta, \gamma) \quad:=\left\{H(\underline{w}) \in \mathcal{W}_{o p}^{\mu, s}|| E \mid \leq \alpha,\right. \\
&\left.\sup _{r \in[0, \infty)}\left|T^{\prime}[r]-1\right| \leq \beta,\left\|\underline{w}_{1}\right\|_{\mu, s, \xi} \leq \gamma\right\},
\end{aligned}
$$

for appropriate $\alpha, \beta, \gamma>0$. Here $H(\underline{w})=E+T+W$, where $E, T$ and $W$ are given in (III.20) and $\underline{w}_{1}:=\left(w_{m, n}\right)_{m+n \geq 1}$.

Lemma IV.1. Fix $0<\rho<1, \mu>0, s \geq 1$, and $0<\xi<1$. Then it follows that the polydisc $\mathcal{D}^{\mu, s}(\rho / 8,1 / 8, \rho / 8)$ is in the domain of the Feshbach map $F_{\rho}$. 
Proof. Let $H(\underline{w}) \in \mathcal{D}^{\mu, s}(\rho / 8,1 / 8, \rho / 8)$. We remark that $W:=H(\underline{w})-E-T$ defines a bounded operator on $\mathcal{F}$, and we only need to check the invertibility of $H(\underline{w})_{\tau \chi_{\rho}}$ on $\operatorname{Ran} \bar{\chi}_{\rho}$. Now the operator $E+T=W_{0,0}[\underline{w}]$ is invertible on $\operatorname{Ran} \bar{\chi}_{\rho}$ since for all $r \in[3 \rho / 4, \infty)$

$$
\begin{aligned}
\operatorname{Re} T[r]+\operatorname{Re} E & \geq r-|T[r]-r|-|E| \\
& \geq r\left(1-\sup _{r}\left|T^{\prime}[r]-1\right|\right)-|E| \\
& \geq \frac{3 \rho}{4}(1-1 / 8)-\frac{\rho}{8} \geq \frac{\rho}{2}
\end{aligned}
$$

and $T:=T\left[H_{f}\right]$. Eqn (IV.4) implies also that $\left\|(E+T)^{-1}\right\| \leq 2 / \rho$. On the other hand, by (III.11), $\|W\| \leq \xi \rho / 8 \leq \rho / 8$. Hence $\left\|\bar{\chi}_{\rho} W \bar{\chi}_{\rho}(E+T)^{-1}\right\| \leq 1 / 4$ and therefore $H(\underline{w})_{\tau, \bar{\chi}_{\rho}}=\left[1+\bar{\chi}_{\rho} W \bar{\chi}_{\rho}(E+T)^{-1}\right](E+T)$ is invertible on $\operatorname{Ran} \bar{\chi}_{\rho}$.

The last part of the proof above gives the estimate

$$
\left\|\left(H(\underline{w})_{\tau \chi_{\rho}}\right)^{-1}\right\| \leq \frac{8}{3 \rho} .
$$

We introduce the scaling transformation $S_{\rho}: \mathcal{B}[\mathcal{F}] \rightarrow \mathcal{B}[\mathcal{F}]$, by

$$
S_{\rho}(\mathbf{1}):=\mathbf{1}, \quad S_{\rho}\left(a^{\#}(k)\right):=\rho^{-3 / 2} a^{\#}\left(\rho^{-1} k\right),
$$

where $a^{\#}(k)$ is either $a(k)$ or $a^{*}(k)$ and $k \in \mathbb{R}^{3}$. On the domain of the decimation map $F_{\rho}$ we define the renormalization map $\mathcal{R}_{\rho}$ as

$$
\mathcal{R}_{\rho}:=\rho^{-1} S_{\rho} \circ F_{\rho}
$$

Remark IV.2. The renormalization map above is different from the one defined in [2]]. The map in [2] contains an additional change of the spectral parameter $\lambda:=-\langle H\rangle_{\Omega}$.

We mention here some properties of the scaling transformation. It is easy to check that $S_{\rho}\left(H_{f}\right)=\rho H_{f}$, and hence

$$
S_{\rho}\left(\chi_{\rho}\right)=\chi_{1} \quad \text { and } \quad \rho^{-1} S_{\rho}\left(H_{f}\right)=H_{f}
$$

which means that the operator $H_{f}$ is a fixed point of $\rho^{-1} S_{\rho}$. Further note that $E \cdot \mathbf{1}$ is expanded under the scaling map, $\rho^{-1} S_{\rho}(E \cdot \mathbf{1})=\rho^{-1} E \cdot \mathbf{1}$, at a rate $\rho^{-1}$. (To control this expansion it is necessary to suitably restrict the spectral parameter.)

Next, we show that the interaction $W$ contracts under the scaling transformation. To this end we remark that the scaling map $S_{\rho}$ restricted to $\mathcal{W}_{o p}^{\mu, s}$ induces a scaling map $s_{\rho}$ on $\mathcal{W}^{\mu, s}$ by

$$
\rho^{-1} S_{\rho}(H(\underline{w}))=: H\left(s_{\rho}(\underline{w})\right) \text {. }
$$


It is easy to verify that $s_{\rho}(\underline{w}):=\left(s_{\rho}\left(w_{m, n}\right)\right)_{m+n \geq 0}$ and, for all $(m, n) \in \mathbb{N}_{0}^{2}$,

$$
s_{\rho}\left(w_{m, n}\right)\left[r, k_{(m, n)}\right]=\rho^{m+n-1} w_{m, n}\left[\rho r, \rho k_{(m, n)}\right] \text {. }
$$

We note that by Theorem III.1, the operator norm of $W_{m, n}\left[s_{\rho}\left(w_{m, n}\right)\right]$ is controlled by the norm

$$
\begin{aligned}
\left\|s_{\rho}\left(w_{m, n}\right)\right\|_{\mu} & =\max _{j} \sup _{r \in I, k \in B_{1}^{m+n}} \rho^{m+n-1} \frac{\left|w_{m, n}\left[\rho r, \rho k_{(m, n)}\right]\right|}{\left|k_{j}\right|^{\mu}} \\
& \leq \rho^{m+n+\mu-1}\left\|w_{m, n}\right\|_{\mu} .
\end{aligned}
$$

Hence, for $m+n \geq 1$, we have that

$$
\left\|s_{\rho}\left(w_{m, n}\right)\right\|_{\mu} \leq \rho^{\mu}\left\|w_{m, n}\right\|_{\mu}
$$

Since $\mu>0$, this estimate shows that $S_{\rho}$ contracts $\left\|w_{m, n}\right\|_{\mu}$ by at least a factor of $\rho^{\mu}<1$. The next result shows that this contraction is actually a property of the renormalization map $\mathcal{R}_{\rho}$ along the 'stable' directions. Recall, $\chi_{1}$ is the cut-off function introduced at the beginning of Section III. Define the constant

$$
C_{\chi}:=\frac{4}{3}\left(\sum_{n=0}^{s} \sup \left|\partial_{r}^{n} \chi_{1}\right|+\sup \left|\partial_{r} \chi_{1}\right|^{2}\right) \leq 200
$$

Clearly, for, say, $s=1, C_{\chi} \geq 4 / 3$. We keep the constant $C_{\chi}$ below in order to relate the analysis of this paper to that of [2].

Theorem IV.3. Let $\epsilon_{0}: H \rightarrow\langle H\rangle_{\Omega}$ and $\mu>0$. Then for the absolute constant $C_{\chi}$ given in (IV.12) and for any $s \geq 1,0<\rho<1 / 2, \alpha, \beta \leq \frac{\rho}{8}$ and $\gamma \leq \frac{\rho}{8 C_{\chi}}$ we have that

$$
\mathcal{R}_{\rho}-\rho^{-1} \epsilon_{0}: \mathcal{D}^{\mu, s}(\alpha, \beta, \gamma) \rightarrow \mathcal{D}^{\mu, s}\left(\alpha^{\prime}, \beta^{\prime}, \gamma^{\prime}\right)
$$

continuously, with $\xi:=\frac{\sqrt{\rho}}{4 C_{\chi}}$ (in the definition of the polydiscs, see (IV.3) and

$$
\alpha^{\prime}=3 C_{\chi}\left(\gamma^{2} / 2 \rho\right), \beta^{\prime}=\beta+3 C_{\chi}\left(\gamma^{2} / 2 \rho\right), \gamma^{\prime}=256 C_{\chi}^{2} \rho^{\mu} \gamma
$$

With some modifications, this theorem follows from Theorem 3.8 in [2] and its proof; especially Equations (3.104), (3.107) and (3.109). For the sake of completeness, we present a proof of this theorem in Appendix I.

Remark IV.4. Subtracting the term $\rho^{-1} \epsilon_{0}$ from $\mathcal{R}_{\rho}$ allows us to control the expanding direction during the iteration of the map $\mathcal{R}_{\rho}$. In [2] such control was achieved by using a change of the spectral parameter $\lambda$, which controls $\langle H\rangle_{\Omega}$. 


\section{Renormalization Group}

In this section we describe some dynamical properties of iterations, $\mathcal{R}_{\rho}^{n} \forall n \geq 1$, of the renormalization map $\mathcal{R}_{\rho}$. A closely related iteration scheme is used in [2]. First, we observe that

$$
\forall \tau \in \mathbb{C}, \mathcal{R}_{\rho}\left(\tau H_{f}\right)=\tau H_{f} \text { and } \mathcal{R}_{\rho}(\tau \mathbf{1})=\frac{1}{\rho} \tau \mathbf{1}
$$

Hence we define $\mathcal{M}_{f p}:=\mathbb{C} H_{f}$ and $\mathcal{M}_{u}:=\mathbb{C} \mathbf{1}$ as candidates for the manifold of fixed points of $\mathcal{R}_{\rho}$ and the unstable manifold. The next result identifies the stable manifold of $\mathcal{M}_{f p}$ which turns out to be of (complex) codimension 1 and is foliated by (complex) codimension 2 stable manifolds, for each fixed point in $\mathcal{M}_{f p}$. This implies, in particular, that, in a vicinity of $\mathcal{M}_{f p}$, there are no other fixed points, and that $\mathcal{M}_{u}$ is the entire unstable manifold of $\mathcal{M}_{f p}$ (see the figure on page 5).

We introduce some definitions. Recall that $D(\lambda, r):=\{z \in \mathbb{C}|| z-\lambda \mid \leq r\}$, a disc in the complex plane. As an initial set of operators we take

$$
\mathcal{D}:=\mathcal{D}^{\mu, s^{\prime}}\left(\alpha_{0}, \beta_{0}, \gamma_{0}\right)
$$

with $\alpha_{0}, \beta_{0}, \gamma_{0} \ll 1$ and $s^{\prime} \geq 1$. We also let

$$
\mathcal{D}_{s}:=\mathcal{D}^{\mu, s^{\prime}}\left(0, \beta_{0}, \gamma_{0}\right)
$$

(The subindex $s$ stands for 'stable', not to be confused with the smoothness index $s$, which, in this section, is denoted $s^{\prime}$.) For $H \in \mathcal{D}$ we write

$$
H_{u}:=\langle H\rangle_{\Omega} \text { and } H_{s}:=H-\langle H\rangle_{\Omega} \mathbf{1}
$$

(the unstable- and stable-central-space components of $H$, respectively). Note that $H_{s} \in$ $\mathcal{D}_{s}$.

We fix the scale $\rho$ so that

$$
\alpha_{0}, \beta_{0}, \gamma_{0} \ll \rho \leq 1 / 2
$$

Below, we use the $n$-th iteration of the numbers $\alpha_{0}, \beta_{0}$ and $\gamma_{0}$ under the map (IV.14):

$$
\begin{gathered}
\alpha_{n}:=c \rho^{-1}\left(c \rho^{\mu}\right)^{2(n-1)} \gamma_{0}^{2} \\
\beta_{n}=\beta_{0}+\frac{c \gamma_{0}^{2}}{\rho} \sum_{j=0}^{n-1}\left(c \rho^{\mu}\right)^{2 j} \\
\gamma_{n}=\left(c \rho^{\mu}\right)^{n} \gamma_{0} .
\end{gathered}
$$


Recall that a vector-function $f$ from an open set $\mathcal{D}$ in a complex Banach space $\mathcal{B}_{1}$ into a complex Banach space $\mathcal{B}_{2}$ is said to be analytic iff $\forall H \in \mathcal{D}$ and $\forall \xi \in \mathcal{B}_{1}, f(H+$ $\tau \xi$ ) is analytic in the complex variable $\tau$ for $|\tau|$ sufficiently small (see [10]). One can show that $f$ is analytic iff it is Gâteaux-differentiable ([10, 21]). A stronger notion of analyticity, requiring in addition that $f$ is locally bounded, is used in [21]. Furthermore, if $f$ is analytic in $\mathcal{D}$ and $g$ is an analytic vector-function from an open set $\Omega$ in $\mathbb{C}$ into $\mathcal{D}$, then the composite function $f \circ g$ is analytic on $\Omega$. In what follows $\mathcal{B}_{1}$ is the space of $H_{f}$-bounded operators on $\mathcal{F}$ and $\mathcal{B}_{2}$ is either $\mathbb{C}$ or $\mathcal{B}(\mathcal{F})$.

For a Banach space $X$ the symbol $O_{X}(\alpha)$ will stand for an element of $X$ bounded in its norm by const $\alpha$.

Theorem V.1. Let $\delta_{n}:=\nu_{n} \rho^{n}$ with $4 \alpha_{n} \leq \nu_{n} \leq \frac{1}{18}$. There is an analytic map e: $\mathcal{D}_{s} \rightarrow$ $D\left(0,4 \alpha_{0}\right)$ s.t. $e(H) \in \mathbb{R}$ for $H=H^{*}$, and

$$
U_{\delta_{n}} \subset D\left(\mathcal{R}_{\rho}^{n}\right) \text { and } \mathcal{R}_{\rho}^{n}\left(U_{\delta_{n}}\right) \subset \mathcal{D}^{\mu, s^{\prime}}\left(\rho / 8, \beta_{n}, \gamma_{n}\right)
$$

where $U_{\delta}:=\left\{H \in \mathcal{D}|| e\left(H_{s}\right)+H_{u} \mid \leq \delta\right\}$. Moreover, $\forall H \in U_{\delta_{n}}$ and $\forall n \geq 1$, there are $E_{n} \in \mathbb{C}$ and $\tau_{n}(r) \in \mathbb{C}$ s.t. $\left|E_{n}\right| \leq 2 \nu_{n},\left|\tau_{n}(r)-1\right| \leq \beta_{n}$, $\tau_{n}$ is $C^{s^{\prime}}$,

$$
\mathcal{R}_{\rho}^{n}(H)=E_{n}+\tau_{n}\left(H_{f}\right) H_{f}+O_{\mathcal{W}_{o p}^{\mu, s^{\prime}}}\left(\gamma_{n}\right),
$$

(the spaces $\mathcal{W}_{o p}^{\mu, s^{\prime}}$ are defined in Section [III), $E_{n}$ and $\tau_{n}(r)$ are real if $H$ is self-adjoint and, as $n \rightarrow \infty, \tau_{n}(r)$ converge in $L^{\infty}$ to some number (constant function) $\tau \in \mathbb{C}$.

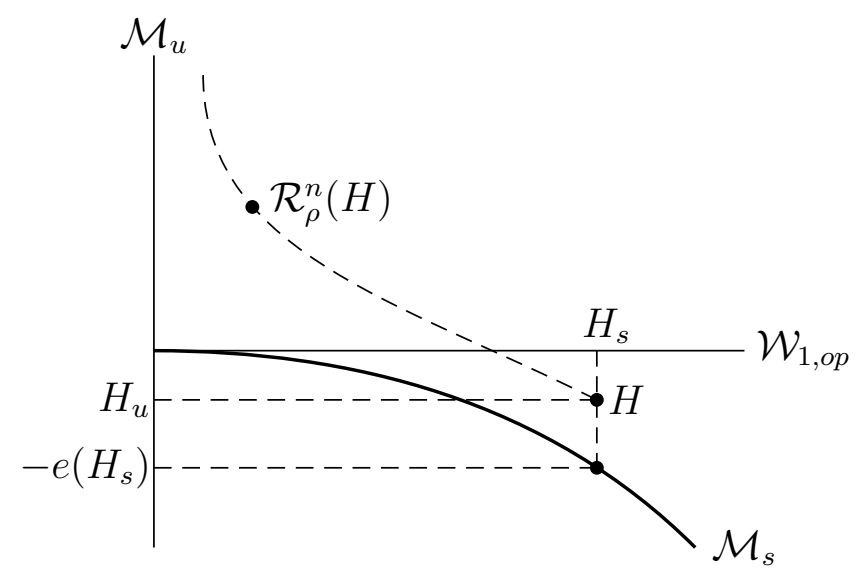

This theorem implies that $\mathcal{M}_{f p}:=\mathbb{C} H_{f}$ is (locally) a manifold of fixed points of $\mathcal{R}_{\rho}$ and $\mathcal{M}_{u}:=\mathbb{C} 1$ is the unstable manifold, and the set

$$
\mathcal{M}_{s}:=\bigcap_{n} U_{\delta_{n}}=\left\{H \in \mathcal{D} \mid e\left(H_{s}\right)=-H_{u}\right\}
$$


is a local stable manifold for the fixed point manifold $\mathcal{M}_{f p}$ in the sense that, $\forall H \in$ $\mathcal{M}_{s}, \exists \tau \in \mathbb{C}$ s.t.

$$
\mathcal{R}_{\rho}^{n}(H) \rightarrow \tau H_{f} \text { in the norm of } \mathcal{W}_{o p}^{\mu, s^{\prime}}
$$

as $n \rightarrow \infty$. Moreover, $\mathcal{M}_{s}$ is an invariant manifold for $\mathcal{R}_{\rho}: \mathcal{M}_{s} \subset D\left(\mathcal{R}_{\rho}\right)$ and $\mathcal{R}_{\rho}\left(\mathcal{M}_{s}\right) \subset$ $\mathcal{M}_{s}$, though we do not need this property here and thus we will not prove it.

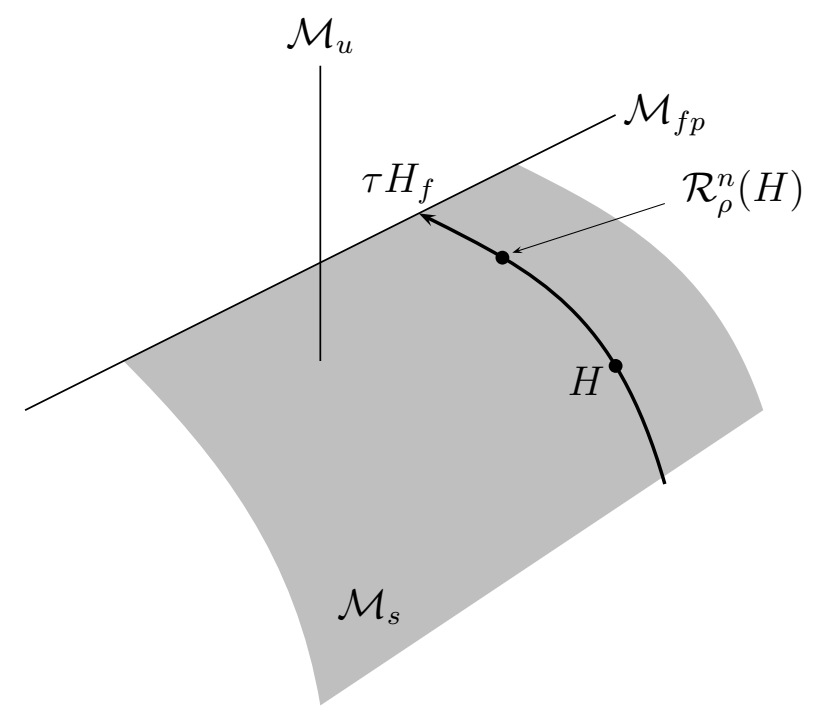

The next result reveals the spectral significance of the map $e$ :

Theorem V.2. Let $H_{s} \in \mathcal{D}_{s}$. Then the number $e\left(H_{s}\right)$ is an eigenvalue of the operator $H_{s}$ and $\sigma\left(H_{s}\right) \subset e\left(H_{s}\right)+S$ where

$$
S:=\left\{w \in \mathbb{C}|\operatorname{Re} w \geq 0,| \operatorname{Im} w \mid \leq \frac{1}{3} \operatorname{Re} w\right\} .
$$

This theorem implies Theorem I.1 formulated in the introduction. We begin with some preliminary results, collected in Proposition $\overline{V .3}$ below, from which we derive Theorems V.1 and V.2.

Proposition V.3. Let $V_{-1} \equiv \mathcal{D}$ and $e_{-1}\left(H_{s}\right)=0 \forall H_{s}$. The triples $\left(V_{n}, E_{n}, e_{n}\right), n=$ $0,1, \ldots$, where $V_{n}$ is a subset of $\mathcal{D}, E_{n}$ is a map of $V_{n-1}$ into $\mathbb{C}$, and $e_{n}$ is a map of $\mathcal{D}_{s}$ into $\mathbb{C}$, are defined inductively in $n \geq 0$ by the formulae

$$
\begin{gathered}
V_{n}:=\left\{H \in \mathcal{D}|| H_{u}+e_{n-1}\left(H_{s}\right) \mid \leq \frac{1}{12} \rho^{n+1}\right\}, \\
E_{n}(H):=\left(\mathcal{R}_{\rho}^{n}(H)\right)_{u}
\end{gathered}
$$




$$
e_{n}\left(H_{s}\right) \text { is the unique zero of the function } E_{n}\left(H_{s}-\lambda\right)
$$

in the disc $D\left(e_{n-1}\left(H_{s}\right), \frac{1}{12} \rho^{n+1}\right)$. Moreover, these objects have the following properties:

$$
V_{n} \subset V_{n-1} \text { and } V_{n} \subset D\left(\mathcal{R}_{\rho}^{n+1}\right),
$$

$E_{n}\left(H_{s}-\lambda\right)$ is analytic in $\lambda \in D\left(e_{n-1}\left(H_{s}\right), \frac{1}{12} \rho^{n+1}\right)$ and in $H_{s} \in \mathcal{D}_{s}, e_{n}\left(H_{s}\right) \in \mathbb{R}$, if $H=H^{*}$, and

$$
\left|e_{n}\left(H_{s}\right)-e_{n-1}\left(H_{s}\right)\right| \leq 2 \alpha_{n} \rho^{n} .
$$

Proof. We proceed by induction in the index $n$. For $n=0$ the proposition is trivially true. We assume that the statements of the proposition hold for all $0 \leq n \leq j-1$ and prove them for $n=j$. Let $e_{n}\left(H_{s}\right)$ and $E_{n}\left(H_{s}-\lambda\right), 0 \leq n \leq j-1$, be as defined in the proposition. Since $e_{j-1}\left(H_{s}\right)$ is defined by ( $(\overline{\mathrm{V} .9})$ with $n=j-1$ we can define $V_{j}$ using (V.7) with $n=j$. Next, by (V.10) with $n=j-1, V_{j-1} \subset D\left(\mathcal{R}_{\rho}^{j}\right)$ and therefore the map $E_{j}$ is well defined.

Let $H \in V_{j-1}$ and denote $\lambda:=-H_{u}$ so that $H:=H_{s}-\lambda$. Let $H^{(j)}(\lambda):=\mathcal{R}_{\rho}^{j}\left(H^{(0)}(\lambda)\right)$ with $H^{(0)}(\lambda):=H_{s}-\lambda$ (we suppress the dependence of $H^{(j)}(\lambda)$ on $H_{s}$ ). Write inductively $H^{(j)}(\lambda):=\mathcal{R}_{\rho}\left(H^{(j-1)}(\lambda)\right)$.

We claim that $H^{(j)}(\lambda)$ is analytic (in the sense specified in the paragraph preceding Theorem V.1) in $\lambda \in D\left(e_{j-1}\left(H_{s}\right), \frac{1}{12} \rho^{j+1}\right)$ and in $H_{s} \in \mathcal{D}_{s}$. We prove this statement by induction in $j$. Clearly, $H^{(0)}(\lambda)=H_{s}-\lambda$ is analytic in $\lambda \in D\left(e_{-1}\left(H_{s}\right), \frac{1}{12} \rho\right)$ and in $H_{s} \in \mathcal{D}_{s}$. Now, assume that $H^{(j-1)}(\lambda)$ is analytic in $\lambda \in D\left(e_{j-2}\left(H_{s}\right), \frac{1}{12} \rho^{j}\right)$ and in $H_{s} \in \mathcal{D}_{s}$. Then by Proposition VIII.1, Appendix III, $H_{0}^{(j-1)}(\lambda):=E^{(j-1)}(\lambda)+T^{(j-1)}(\lambda)$ and $W^{(j-1)}(\lambda)$ are analytic. By the properties of $T^{(j-1)}(\lambda)$, the inverse $H_{0}^{(j-1)}(\lambda)^{-1} \bar{\chi}_{\rho}$ is well-defined and is analytic and therefore so is

$$
\bar{\chi}_{\rho} H^{(j-1)}(\lambda)_{\tau, \bar{\chi}_{\rho}}^{-1} \bar{\chi}_{\rho}=\sum_{n=0}^{\infty} \bar{\chi}_{\rho}\left(-H_{0}^{(j-1)}(\lambda)^{-1} \bar{\chi}_{\rho} W^{(j-1)}(\lambda) \bar{\chi}_{\rho}\right)^{n} H_{0}^{(j-1)}(\lambda)^{-1} \bar{\chi}_{\rho} .
$$

By the definition of the decimation map, (IV.1)-(IV.2),

$$
F_{\rho}\left(H^{(j-1)}(\lambda)\right)=H_{0}^{(j-1)}(\lambda)+\chi_{\rho} W^{(j-1)}(\lambda) \bar{\chi}_{\rho} H^{(j-1)}(\lambda)_{\tau, \bar{\chi}_{\rho}}^{-1} \bar{\chi}_{\rho} W^{(j-1)}(\lambda) \chi_{\rho},
$$

is analytic. Hence, by the definition of the renormalization map $\mathcal{R}_{\rho}$ in (IV.6) - (IV.7), $\mathcal{R}_{\rho}\left(H^{(j-1)}(\lambda)\right)$ is analytic as well.

This implies that $E_{j}\left(H_{s}-\lambda\right)$ is analytic in $\lambda \in D\left(e_{j-1}, \frac{1}{12} \rho^{j+1}\right)$ and in $H_{s} \in \mathcal{D}_{s}$.

In the remaining part of the proof we will use the shorthand $e_{n} \equiv e_{n}\left(H_{s}\right)$ and (abusing notation) $E_{n}(\lambda) \equiv E_{n}\left(H_{s}-\lambda\right)$. Now, we prove ( (V.9) and ( (V.11) with $n=j$. We begin with some preliminary estimates. Let $H \in V_{j-1}$. For $1 \leq n \leq j$ denote

$$
\Delta_{n} E(\lambda):=E_{n}(\lambda)-\rho^{-1} E_{n-1}(\lambda) .
$$


Since $\mathcal{R}_{\rho}^{n}(H)=\mathcal{R}_{\rho}\left(\mathcal{R}_{\rho}^{n-1}(H)\right)$, we have, by Theorem IV.3, that $\left|\Delta_{n} E(\lambda)\right| \leq \alpha_{n}$. This and the analyticity of $\Delta_{n} E(\lambda)$ in $D\left(e_{n-1}, \frac{1}{12} \rho^{n+1}\right)$ together with the Cauchy formula imply that

$$
\left|\partial_{\lambda}^{m} \Delta_{n} E(\lambda)\right| \leq \alpha_{n}\left(\frac{1}{12} \rho^{n+1}\right)^{-m} \text { for } n \leq j \text { and } m=0,1 .
$$

Iterating (V.12) we find for $i \leq j$

$$
E_{i}(\lambda)=\rho^{-i}\left(E_{0 i}(\lambda)-\lambda\right)
$$

where

$$
E_{0 i}(\lambda):=\sum_{n=1}^{i} \rho^{n} \Delta_{n} E(\lambda)
$$

By the estimate (V.13) with $m=1$ we have for $i \leq j$

$$
\left|\partial_{\lambda} E_{0 i}(\lambda)\right| \leq \sum_{n=1}^{i} \rho^{n}\left|\partial_{\lambda} \Delta_{n} E(\lambda)\right| \leq c \sum_{n=1}^{i} c^{2 n-1} \rho^{2 \mu(n-1)-2} \gamma_{0}^{2}
$$

which, by the conditions on the parameters, (V.1), implies

$$
\left|\partial_{\lambda} E_{0 i}(\lambda)\right| \leq c \rho^{-2} \gamma_{0}^{2} \leq \frac{1}{5}
$$

for $0<i \leq j$.

Now, we are ready to show the existence and properties of $e_{j}$, stated in (V.9) and (V.11) with $n=j$, i.e. to show that $E_{j}(\lambda)$ has a unique zero, $e_{j}$, in every disc $D\left(e_{j-1}, r \rho^{j}\right)$ with $2 \alpha_{j} \leq r \leq \frac{1}{12} \rho$. The latter is equivalent to showing that $e_{j}$ is a fixed point of the map $\lambda \rightarrow E_{0 j}(\lambda)$ in the discs $D\left(e_{j-1}, r \rho^{j}\right)$. Using the equations $e_{j-1}=E_{0 j-1}\left(e_{j-1}\right)$ and (V.15) with $i=j-1, j$ and using the triangle inequality we obtain

$$
\left|E_{0 j}(\lambda)-e_{j-1}\right| \leq \rho^{j}\left|\Delta_{j} E(\lambda)\right|+\left|E_{0 j-1}(\lambda)-E_{0 j-1}\left(e_{j-1}\right)\right|
$$

Now, remembering the estimate $(\overline{\mathrm{V} .13})$ (with $m=0$ and $n=j$ ) and the estimate (V.16) (with $i=j-1$ ) and using the mean-value theorem we arrive at the inequality

$$
\left|E_{0 j}(\lambda)-e_{j-1}\right| \leq \rho^{j} \alpha_{j}+\frac{1}{5}\left|\lambda-e_{j-1}\right|
$$

and therefore, $\left|E_{0 j}(\lambda)-e_{j-1}\right| \leq r \rho^{j}$, provided $\left|\lambda-e_{j-1}\right| \leq r \rho^{j}$ (remember that $\alpha_{j} \leq$ $\left.\alpha_{0} \ll \rho \ll 1\right)$. This inequality together with Eqn (V.16) with $i=j$ implies that the map $\lambda \rightarrow E_{0 j}(\lambda)$ has a unique fixed point, $e_{j}$, in the disc $D\left(e_{j-1}, r \rho^{j}\right)$. For $r=\frac{1}{12} \rho$ this gives (V.9) with $n=j$. Taking $r=2 \alpha_{j}$ we arrive at (V.11) with $n=j$. 
If $H$ is self-adjoint, then so is the operator $\mathcal{R}_{\rho}(H)$, and, consequently, $\mathcal{R}_{\rho}^{j}(H)=$ $\mathcal{R}_{\rho}^{j}(H)^{*}$. Hence $E_{j}(\lambda)$ and $e_{j}$ are real in this case.

Next, we show the first inclusion in (V.10) for $n=j$. Let $H \in V_{j}$ and hence $\mid \lambda-$ $e_{j-1} \mid \leq \frac{1}{12} \rho^{j+1}$. Then, by the induction assumption (V.11) for $n=j-1$, we have that $\left|\lambda-e_{j-2}\right| \leq \frac{1}{12} \rho^{j+1}+2 \alpha_{j-1} \rho^{j-1} \leq \frac{1}{12} \rho^{j}$ and therefore $H \in V_{j-1}$, as claimed.

We proceed to show the second inclusion in ( $(\mathrm{V} .10)$ for $n=j$. Let $H \in V_{j}$ and keep the notation as above. Since $E_{j-1}\left(e_{j-1}\right)=0$, we have that $\left|E_{j}(\lambda)\right| \leq\left|\Delta_{j} E(\lambda)\right|+$ $\rho^{-1}\left|E_{j-1}(\lambda)-E_{j-1}\left(e_{j-1}\right)\right|$ which by (V.13), (V.14) and (V.16) with $i=j-1$ gives $\left|E_{j}(\lambda)\right| \leq \alpha_{j}+\frac{6}{5} \rho^{-j}\left|\lambda-e_{j-1}\right|$. Hence, since $\alpha_{j} \leq \alpha_{0}$ and by (V.1),

$$
\left|E_{j}(\lambda)\right| \leq \frac{1}{8} \rho
$$

provided $\left|\lambda-e_{j-1}\right| \leq \frac{1}{12} \rho^{j+1}$. Thus, using Theorem IV.3 and (V.18) we conclude that, for $n:=j$,

$$
\mathcal{R}_{\rho}^{n}\left(V_{n}\right) \subset \mathcal{D}^{\mu, 1}\left(\rho / 8, \beta_{n}, \gamma_{n}\right)
$$

with the numbers $\beta_{n}$ and $\gamma_{n}$ given inductively by $\beta_{n}=\beta_{n-1}+3 C_{\chi} \frac{\gamma_{n-1}^{2}}{2 \rho}$ and $\gamma_{n}=$ $256 C_{\chi}^{2} \rho^{\mu} \gamma_{n-1}$ and in final form, in the paragraph preceding Theorem VI.1. Clearly, $\beta_{n}$, $\gamma_{n} \leq \frac{\rho}{8}$. E.g. $\beta_{n} \leq \beta_{0}+c \frac{\gamma_{0}^{2}}{\rho}\left(1-\left(c \rho^{\mu}\right)^{2}\right)^{-1}<\frac{\rho}{8}$. Hence, by Lemma IV.1 $\mathcal{R}_{\rho}^{j}\left(V_{j}\right) \subset$ $D\left(\mathcal{R}_{\rho}\right)$. Thus $(\mathrm{V} .10)$ is proven for $n=j$.

Proof of Theorem V.1 By (V.11), the limit $e\left(H_{s}\right):=\lim _{j \rightarrow \infty} e_{j}\left(H_{s}\right)$ exists pointwise for $H \in \mathcal{D}$. Iterating Eqn (V.11) we find the estimate

$$
\left|e_{n}\left(H_{s}\right)-e\left(H_{s}\right)\right| \leq 3 \alpha_{n+1} \rho^{n+1}
$$

Given that $\alpha_{0} \leq \frac{\rho}{108}$ (this is a condition on the (bare) coupling constant $g$ ), this inequality implies that

$$
V_{n} \subset U_{\delta_{n}} \subset V_{n-1}
$$

where $\delta_{n}:=\frac{1}{18} \rho^{n}$.

To prove the analyticity of $e\left(H_{s}\right)$ we note that, since $E_{j}\left(\lambda, H_{s}\right)$ is analytic in $H_{s} \in \mathcal{D}_{s}$, then so is $e_{j}\left(H_{s}\right)$. By (V.11) the limit $e\left(H_{s}\right):=\lim _{j \rightarrow \infty} e_{j}\left(H_{s}\right)$ is also analytic in $H_{s} \in \mathcal{D}_{s}$.

Eqns ( $(\mathrm{V} .10)$ and $(\overline{\mathrm{V} .21})$ imply the first part of ( $\mathrm{V} .2)$. The second part of ( $(\mathrm{V} .2)$ follows from Theorem IV.3 and (V.18).

Now we prove the last statement of Theorem V.1. Let $H \in U_{\delta_{n}} \subset V_{n} \subset D\left(\mathcal{R}_{\rho}^{n+1}\right)$. According to (III.19), $H^{(n)}:=\mathcal{R}_{\rho}^{n}(H)$ can be written as

$$
H^{(n)}=E_{n} \mathbf{1}+T_{n}+W_{n}
$$


where $T_{n} \equiv T_{n}\left(H_{f}\right)$ with $T_{n}(r) \in C^{1}$ and $T_{n}(0)=0$. Hence the function $\tau_{n}(r):=$ $T_{n}(r) / r$ is well defined. By (V.19) we have $\left|\partial_{r} T_{n}(r)-1\right| \leq \beta_{n}$ and $\left\|W_{n}\right\|_{\mathcal{W}_{o p}^{s}} \leq \gamma_{n}$. This gives the desired estimates for the last two terms in ( (V.3). Let $E_{n}(\lambda) \equiv E_{n}$ for $\lambda:=-H_{u}$. To prove the bound on the first term on the r.h.s. of ( $\mathrm{V} .3)$ we use the relation $E_{n}\left(e_{n}\right)=0$ and Eqns (V.14) and (V.16) to obtain

$$
\left|E_{n}(\lambda)\right|=\left|E_{n}(\lambda)-E_{n}\left(e_{n}\right)\right| \leq \frac{6}{5} \rho^{-n}\left|\lambda-e_{n}\right|
$$

This inequality together with (V.20) implies, $\left|E_{n}(\lambda)\right| \leq \frac{6}{5} \nu_{n}+\frac{18}{5} \alpha_{n+1} \rho \leq 2 \nu_{n}$, provided $|\lambda-e| \leq \nu_{n} \rho^{n}$ and $4 \alpha_{n} \leq \nu_{n}$. Finally, if $H$ is self-adjoint, then so is $\mathcal{R}_{\rho}^{n}(H)$ and therefore $E_{n}$ and $\tau_{n}(r):=T_{n}(r) / r$ are real.

To complete the proof of Theorem $V$.1 it remains to show that as $n \rightarrow \infty$, the functions $\tau_{n}(r)$ converge in $L^{\infty}$ to a constant function, $\tau$, as $n \rightarrow \infty$. To prove this property requires representing the operators $T^{(n)}$ as sums of the $j$-th step corrections,

$$
\Delta_{n} T(r):=T_{n}(r)-\rho^{-1} T_{n-1}(\rho r),
$$

similarly to (V.14) and (V.15). In fact, this analysis gives that $\tau=\lim _{n \rightarrow \infty} \tau_{n}(0)$. We omit the details here but refer the reader to [2].

Proof of Theorem V.2 It is shown in Appendix II (SectionVII), Theorem VII.1, that $e\left(H_{s}\right)$ is an eigenvalue of $H_{s}$ (cf. [7, 8, 2]). Here we show the second statement of the theorem regarding the spectrum of $H_{s}$. As above, we omit the reference to $H_{s}$ and set $e \equiv e\left(H_{s}\right)$ and $e_{n} \equiv e_{n}\left(H_{s}\right)$.

We first consider the case of a self-adjoint operator $H_{s}$. Let $H^{(n)}(\lambda):=\mathcal{R}_{\rho}^{n}\left(H_{s}-\lambda\right)$ and, recall, $E_{n}(\lambda):=H^{(n)}(\lambda)_{u}$. Eqns (V.14) and (V.16) imply the estimate $\partial_{\lambda} E_{n}(\lambda) \leq$ $-\frac{4}{5} \rho^{-n}$. Using the equation $E_{n}\left(e_{n}\right)=0$, the mean value theorem and the estimate above, we obtain that $E_{n}(\lambda) \geq-\frac{4}{5} \rho^{-n}\left(\lambda-e_{n}\right)$, provided $\lambda \leq e_{n}$. Hence, if $\lambda \leq e_{n}-\theta_{n}$, with $\theta_{n} \gg \gamma_{n} \rho^{n}$ and $\theta_{n} \rightarrow 0$ as $n \rightarrow \infty$, then $H^{(n)}(\lambda) \geq \frac{4}{5} \rho^{-n} \theta_{n}-O\left(\gamma_{n}\right) \geq \frac{1}{2} \gamma_{n}$. This implies $0 \in \rho\left(H^{(n)}(\lambda)\right)$ and therefore, by Theorem II.1 $0 \in \rho\left(H_{s}-\lambda\right)$ or $\lambda \in \rho\left(H_{s}\right)$. Since $e_{n} \rightarrow e$ and $\theta_{n} \rightarrow 0$ as $n \rightarrow \infty$, this implies that $\sigma\left(H_{s}\right) \subset[e, \infty)$, which is the second statement of the theorem for self-adjoint operators.

Now we consider a non-self-adjoint operator $H_{s}$. For all $n \geq 0$, we have shown that if $H_{s} \in \mathcal{D}_{s}, e=e\left(H_{s}\right)$ and if $|\lambda-e| \leq \delta_{n}$, where $\delta_{n}=\nu_{n} \rho^{n}$, then $H_{s}-\lambda \in \operatorname{dom}\left(\mathcal{R}_{\rho}^{n}\right)$ and $H^{(n)}(\lambda):=\mathcal{R}_{\rho}^{n}\left(H_{s}-\lambda\right) \in D^{\mu, 1}\left(\rho / 8, \beta_{n}, \gamma_{n}\right)$. By Theorem $\Pi$ we have that

$$
\lambda \in \sigma\left(H_{s}\right) \quad \Leftrightarrow \quad 0 \in \sigma\left(H^{(n)}(\lambda)\right),
$$

if $|\lambda-e| \leq \delta_{n}$. By Theorem V.1, we can decompose

$$
H^{(n)}(\lambda)=E_{n}(\lambda)+\tau_{n}\left(H_{f}, \lambda\right) H_{f}+W_{n}(\lambda),
$$


with $\left\|W_{n}(\lambda)\right\| \leq \gamma_{n}$ on $\operatorname{Ran} \chi_{H_{f} \leq \rho}$. Hence

$$
0 \in \sigma\left(H^{(n)}(\lambda)\right) \Rightarrow \exists r \in[0, \rho]:\left|E_{n}(\lambda)+\tau_{n}(r, \lambda) r\right| \leq \gamma_{n} .
$$

Using that $E_{n}\left(e_{n}\right)=0\left(e_{n} \equiv e_{n}\left(H_{s}\right)\right)$ and the integral of derivative formula we find

$$
E_{n}(\lambda)=\left(\lambda-e_{n}\right) g(\lambda)
$$

with $g(\lambda):=\int_{0}^{1} E_{n}^{\prime}\left(e_{n}+s\left(\lambda-e_{n}\right)\right) d s$. Note that $\bar{\lambda}:=e_{n}+s\left(\lambda-e_{n}\right)$ satisfies $|\bar{\lambda}-e| \leq \delta_{n}$ for $0 \leq s \leq 1$. This and (V.14), (V.16) and $\rho^{-1} \gamma_{0} \ll 1$ imply that

$$
\left|g(\lambda)+\rho^{-n}\right| \leq \frac{1}{5} \rho^{-n} .
$$

In addition, below we use the estimate ( $(\mathbf{V . 2 0})$ which we rewrite as:

$$
\left|e_{n}-e\right| \leq 3 \alpha_{n+1} \rho^{n+1} \text {. }
$$

We denote $\mu:=\lambda-e$ so that

$$
E_{n}(\lambda)=g(\lambda)\left(\mu+e-e_{n}\right)
$$

We consider separately two cases.

a) $\operatorname{Re} \mu \leq-\theta$ and $|\operatorname{Im} \mu| \leq 3 \theta$ with $\theta \geq 36 \alpha_{n+1} \rho^{n+1}$. Using

$$
\operatorname{Re}\left(E_{n}+\tau_{n} r\right)=\operatorname{Re} g \operatorname{Re} \mu-\operatorname{Im} g \operatorname{Im} \mu+\operatorname{Re}\left(g\left(e-e_{n}\right)\right)+\operatorname{Re} \tau_{n} r
$$

and using (V.29), we obtain

$$
\begin{gathered}
\operatorname{Re}\left(E_{n}+\tau_{n} r\right) \geq \frac{4}{5} \rho^{-n} \theta-\frac{3}{5} \rho^{-n} \theta \\
-\frac{6}{5} \rho^{-n} 3 \alpha_{n+1} \rho^{n+1}+\left(1-\beta_{n}\right) r .
\end{gathered}
$$

Since $\theta \geq 36 \alpha_{n+1} \rho^{n+1}$ this gives

$$
\operatorname{Re}\left(E_{n}+\tau_{n} r\right) \geq \frac{1}{10} \rho^{-n} \theta+\left(1-\beta_{n}\right) r .
$$

b) $|\operatorname{Im} \mu| \geq \theta$ and $|\operatorname{Re} \mu| \leq 3 \theta$. If $r \leq 10 \theta \rho^{-n}$, then

$$
\begin{aligned}
\left|\operatorname{Im}\left(E_{n}+\tau_{n} r\right)\right| & =\operatorname{Re} g \operatorname{Im} \mu+\operatorname{Im} g \operatorname{Re} \mu+\operatorname{Im}\left(E_{n}^{\prime}\left(e-e_{n}\right)\right) \\
& +\operatorname{Im} \tau_{n} r \mid \geq \frac{4}{5} \rho^{-n} \theta-\frac{3}{5} \rho^{-n} \theta \\
& -\frac{6}{5} \rho^{-n} 3 \alpha_{n+1} \rho^{n+1}-\beta_{n} 10 \theta \rho^{-n} .
\end{aligned}
$$


This gives

$$
\left|\operatorname{Im}\left(E_{n}+\tau_{n} r\right)\right| \geq \frac{1}{10} \theta \rho^{-n}
$$

provided $\theta \geq 72 \alpha_{n+1} \rho^{n+1}$ and $\beta_{n} \leq 10^{-3}$.

Now, if $r \geq 10 \theta \rho^{-n}$, then we estimate by (V.31) and (V.29)

$$
\left|E_{n}+\tau_{n} r\right| \geq\left|g \mu+\tau_{n} r\right|-\frac{6}{5} \rho^{-n} 3 \alpha_{n+1} \rho^{n+1} .
$$

Furthermore, we have

$$
\begin{aligned}
\left|g \mu+\tau_{n} r\right|^{2} & =\left(\operatorname{Re} g \operatorname{Re} \mu-\operatorname{Im} g \operatorname{Im} \mu+\operatorname{Re} \tau_{n} r\right)^{2} \\
& +\left(\operatorname{Re} g \operatorname{Im} \mu+\operatorname{Im} g \operatorname{Re} \mu+\operatorname{Im} \tau_{n} r\right)^{2} \\
& \geq\left(\operatorname{Re} g \operatorname{Re} \mu+\operatorname{Re} \tau_{n} r\right)^{2}-\left(\frac{1}{5} \rho^{-n} \operatorname{Im} \mu\right)^{2} \\
& +\frac{4}{5} \rho^{-n}|\operatorname{Im} \mu|-\frac{3}{5} \rho^{-n}|\operatorname{Im} \mu|^{2}-\left(\beta_{n} r\right)^{2} .
\end{aligned}
$$

Since $\operatorname{Re} g \operatorname{Re} \mu+\frac{1}{2} \operatorname{Re} \tau_{n} r \geq 0$, we have $\left|g \mu+\tau_{n} r\right|^{2} \geq\left(\frac{1}{2} \operatorname{Re} \tau_{n} r\right)^{2}-\left(\beta_{n} r\right)^{2}$ which gives, for $\theta \leq 10^{-3}$,

$$
\begin{aligned}
\left|g \mu+\tau_{n} r\right| & \geq \frac{1}{2}\left(1-2 \beta_{n}\right) r \\
& \geq 5\left(1-2 \beta_{n}\right) \theta \rho^{-n} \geq 2 \theta \rho^{-n} .
\end{aligned}
$$

This together with (V.34) yields $\left|E_{n}+\tau_{n} r\right| \geq \theta \rho^{-n}$, provided $\theta \geq 4 \alpha_{n+1} \rho^{n+1}$. This together with (V.33) gives for the case b)

$$
\left|E_{n}+\tau_{n} r\right| \geq \frac{1}{10} \theta \rho^{-n}
$$

provided $\theta \geq 72 \alpha_{n+1} \rho^{n+1}$ and $\theta \leq 10^{-3}$.

The inequalities ( $\mathrm{V.32}$ ) and (V.35) and relations (V.25) and (V.27) show that $\lambda \in \rho\left(H_{s}\right)$ if either $\operatorname{Re} \mu \leq-\theta$ and $|\operatorname{Im} \mu| \leq 3 \theta$ or $\operatorname{Im} \mu \geq \theta$ and $|\operatorname{Re} \mu| \leq 3 \theta$ with $\mu:=\lambda-e$, provided

$$
\theta \geq \max \left(20 \rho^{n} \gamma_{n}, 72 \alpha_{n+1} \rho^{n+1}\right) \text { and } \beta_{n} \leq 10^{-3} \text {. }
$$

This can be written as

$$
\Omega_{\theta}^{(1)}, \Omega_{\theta}^{(2)} \subset \rho\left(H_{s}\right)
$$

where $\theta$ satisfies $\mathrm{V.36}$ and

$$
\Omega_{\theta}^{(1)}:=\{\lambda \in \mathbb{C} \mid \operatorname{Re} \mu \leq-\theta \text { and }|\operatorname{Im} \mu| \leq 3 \theta\}
$$


and

$$
\Omega_{\theta}^{(2)}:=\{\lambda \in \mathbb{C}|| \operatorname{Im} \mu \mid \geq \theta \text { and }|\operatorname{Re} \mu| \leq 3 \theta\} .
$$

Define the new subset $\Omega_{\theta}^{(3)}:=\{\lambda \in \mathbb{C} \mid \operatorname{Re} \mu \leq-\theta\}$. We claim that

$$
\Omega_{\theta}^{(3)} \subset \cup_{n=0}^{\infty}\left(\Omega_{3^{n} \theta}^{(1)} \cup \Omega_{3^{n+1} \theta}^{(2)}\right) .
$$

Indeed, $\Omega_{\theta}^{(3)} /\left(\Omega_{\theta}^{(1)} \cup \Omega_{3 \theta}^{(2)}\right) \bigcap \Omega_{\theta}^{(3)} \subset\{\lambda \in \mathbb{C}|\operatorname{Re} \mu \leq-3 \theta,| \operatorname{Im} \mu \mid \geq 3 \theta\} \subset \Omega_{3 \theta}^{(3)}$ and therefore $\Omega_{\theta}^{(3)} \subset \Omega_{\theta}^{(1)} \cup \Omega_{3 \theta}^{(2)} \cup \Omega_{3 \theta}^{(3)}$. Iterating the last inclusion we arrive at the desired relation. Eqns (V.37) and (V.38) imply that

$$
\Omega_{\theta}^{(2)} \cup \Omega_{\theta}^{(3)} \subset \rho\left(H_{s}\right)
$$

for any $\theta$ satisfying (V.36).

Now assume $\lambda \notin e+S$, where $S$ is defined in (V.6). Then either $\operatorname{Re} \mu<0$ or $\operatorname{Re} \mu \geq 0$ and $|\operatorname{Im} \mu|>\frac{1}{3} \operatorname{Re} \mu$. In the first case $\exists n$ s.t. $\operatorname{Re} \mu<-\theta_{n}$ where $\theta_{n}:=$ $\max \left(20 \rho^{n} \delta_{n}, 72 \delta_{n+1} \rho^{n+1}\right)$, and therefore $\lambda \in \Omega_{\theta_{n}}^{(3)} \subset \rho\left(H_{s}\right)$. In the second case, assuming $\mu>0$, we choose $n$ s.t. $\operatorname{Re} \mu \approx 3 \theta_{n}$. Then $|\operatorname{Im} \mu| \geq \theta_{n}$ and $|\operatorname{Re} \mu| \leq 3 \theta_{n}$ so that $\lambda \in \Omega_{\theta_{n}}^{(2)} \subset \rho\left(H_{s}\right)$. Hence $\mathbb{C} /\{e+S\} \subset \rho\left(H_{s}\right)$ which implies $\sigma\left(H_{s}\right) \subset e\left(H_{s}\right)+S$.

Remark V.4. Define $E_{0 \infty}\left(e\left(H_{s}\right), H_{s}\right):=\lim _{j \rightarrow \infty} E_{0 j}\left(e\left(H_{s}\right), H_{s}\right)$. Then

$$
E_{0 \infty}\left(e\left(H_{s}\right), H_{s}\right)=\sum_{i=1}^{\infty} \rho^{i} \Delta_{i} E\left(e\left(H_{s}\right), H_{s}\right)
$$

where the series on the right hand side converges absolutely by estimate ( $(\mathrm{V} .13)$, and $e\left(H_{s}\right)$ satisfies the relation

$$
e\left(H_{s}\right)=E_{0 \infty}\left(e\left(H_{s}\right), H_{s}\right) .
$$

Indeed, Eqns (V.14) and (V.18) yield $\left|E_{0 j}\left(\lambda, H_{s}\right)-\lambda\right| \leq \frac{1}{8} \rho^{j}$, provided $\left|\lambda-e_{j-1}\left(H_{s}\right)\right| \leq$ $\frac{1}{12} \rho^{j+1}$, which together with (V.20) implies (V.40).

Eqns (V.39) - (V.40), (V.14) and (V.13) (with $m=0)$ imply that

$$
\begin{aligned}
\left|E_{0 n}(\lambda)-e\right| & \leq\left|E_{0 n}(\lambda)-E_{0 n}(e)\right|+\left|E_{0 n}(e)-E_{0 \infty}(e)\right| \\
& \leq \sup _{\lambda \in A_{\delta_{n}}}\left(\left|E_{0 n}^{\prime}(\lambda)\right|\right)|\lambda-e|+\sum_{i=n+1}^{\infty} \rho^{i} \alpha_{i} .
\end{aligned}
$$

Now, using Eqn (V.16) and the definition of $\alpha_{i}$ we obtain, furthermore, that

$$
\left|E_{0 n}(\lambda)-e\right| \leq \frac{1}{5}|\lambda-e|+(1-\rho)^{-1} \rho^{n+1} \alpha_{n+1}
$$

This estimate is used in our further work, [17]. 


\section{Appendix I: Proof of Theorem IV.3}

The proof below is similar to and relies on some parts of the corresponding proof in [2]. We proceed in two steps. First we determine $\underline{\hat{w}}$ s.t. $H(\underline{\hat{w}})=: \mathcal{R}_{\rho}(H(\underline{w}))$. In fact, we find explicit formulae expressing $\underline{\hat{w}}$ in terms of $\underline{w}$. Then, using these formulae, we estimate $\underline{\hat{w}}$.

Let $H(\underline{w}) \in \mathcal{D}^{\mu, 0}(\rho / 8,1 / 8, \rho / 8)$. We write this operator as $H(\underline{w})=H_{0}+W$ where $H_{0}:=E+T$. According to the definition (Eqns (III.3) ) and (IIV.2 $)$ ) of the smooth Feshbach map, $F_{\rho}$, we have that

$$
\begin{aligned}
& F_{\rho}(H(\underline{w}))=H_{0}+\chi_{\rho} W \chi_{\rho} \\
& \quad-\chi_{\rho} W \bar{\chi}_{\rho}\left(H_{0}+\bar{\chi}_{\rho} W \bar{\chi}_{\rho}\right)^{-1} \bar{\chi}_{\rho} W \chi_{\rho} .
\end{aligned}
$$

Here, recall, the cut-off operators $\chi_{\rho} \equiv \chi_{H_{f} \leq \rho}$ are defined in Section $\left[\right.$ III and $\bar{\chi}_{\rho}:=1-\chi_{\rho}$. Note that, because of $H(\underline{w}) \in \mathcal{D}^{\mu, 0}(\rho / 8,1 / 8, \rho / 8)$ and of (III.13)

$$
\left\|H_{0}^{-1} \bar{\chi}_{\rho}^{2}\right\| \leq \frac{2}{\rho} \quad \text { and } \quad\|W\| \leq \frac{\xi \rho}{8} .
$$

Eq. (VI.2) implies that the Neumann series expansion in $W_{\bar{\chi}_{\rho}}:=\bar{\chi}_{\rho} W \bar{\chi}_{\rho}$ of the resolvent in (VI.1) is norm convergent and yields

$$
F_{\rho}(H(\underline{w}))=H_{0}+\sum_{L=1}^{\infty}(-1)^{L-1} \chi_{\rho} W\left(H_{0}^{-1} \bar{\chi}_{\rho}^{2} W\right)^{L-1} \chi_{\rho} .
$$

To write the Neumann series on the right side of (VI.3) in the generalized normal form we use Wick's theorem, which we formulate now. We begin with some notation. We introduce the operator families

$$
\begin{aligned}
& W_{p, q}^{m, n}\left[\underline{w} \mid r ; k_{(m, n)}\right]:=\chi_{1} \int_{B_{1}^{p+q}} \frac{d x_{(p, q)}}{\left|x_{(p, q)}\right|^{1 / 2}} a^{*}\left(x_{(p)}\right) \times \\
& w_{m+p, n+q}\left[H_{f}+r ; k_{(m)}, x_{(p)}, \tilde{k}_{(n)}, \tilde{x}_{(q)}\right] a\left(\tilde{x}_{(q)}\right) \chi_{1},
\end{aligned}
$$

for $m+n \geq 0$ and a.e $k_{(m, n)} \in B_{1}^{m+n}$. Here we use the notation for $x_{(p, q)}, x_{(p)}, \tilde{x}_{(q)}$, etc. similar to the one introduced in Eqs. (III.2)-(III.4). For $m=0$ and/or $n=0$, the variables $k_{(0)}$ and/or $\tilde{k}_{(0)}$ are dropped out. Denote by $S_{m}$ the group of permutations of $m$ elements. Define the symmetrization operation as

$$
\begin{aligned}
& w_{m, n}^{(\mathrm{sym})}\left[r ; k_{(m, n)}\right] \\
& \quad:=\frac{1}{m ! n !} \sum_{\pi \in S_{m}} \sum_{\tilde{\pi} \in S_{n}} w_{m, n}\left[r ; k_{\pi(1)}, \ldots, k_{\pi(m)} ; \tilde{k}_{\tilde{\pi}(1)}, \ldots, \tilde{k}_{\tilde{\pi}(n)}\right] .
\end{aligned}
$$


Finally, below we will use the notation

$$
\begin{aligned}
& \Sigma\left[k_{(m)}\right]:=\left|k_{1}\right|+\ldots+\left|k_{m}\right|, \\
& k_{(M, N)}=\left(k_{\left(m_{1}, n_{1}\right)}^{(1)}, \ldots, k_{\left(m_{L}, n_{L}\right)}^{(L)}\right), \quad k_{\left(m_{\ell}, n_{\ell}\right)}^{(\ell)}=\left(k_{\left(m_{\ell}\right)}^{(\ell)}, \tilde{k}_{\left(n_{\ell}\right)}^{(\ell)}\right), \\
& r_{\ell}:=\Sigma\left[\tilde{k}_{\left(n_{1}\right)}^{(1)}\right]+\ldots+\Sigma\left[\tilde{k}_{\left(n_{\ell-1}\right)}^{(\ell-1)}\right]+\Sigma\left[k_{\left(m_{\ell+1}\right)}^{(\ell+1)}\right]+\ldots+\Sigma\left[k_{\left(m_{L}\right)}^{(L)}\right], \\
& \tilde{r}_{\ell}:=\Sigma\left[\tilde{k}_{\left(n_{1}\right)}^{(1)}\right]+\ldots+\Sigma\left[\tilde{k}_{\left(n_{\ell}\right)}^{(\ell)}\right]+\Sigma\left[k_{\left(m_{\ell+1}\right)}^{(\ell+1)}\right]+\ldots+\Sigma\left[k_{\left(m_{L}\right)}^{(L)}\right],
\end{aligned}
$$

with $r_{\ell}=0$ if $n_{1}=\ldots n_{\ell-1}=m_{\ell+1}=\ldots m_{L}=0$ and similarly for $\tilde{r}_{\ell}$ and $m_{1}+\ldots+$ $m_{L}=M, n_{1}+\ldots+n_{L}=N$.

Theorem VI.1 (Wick Ordering). Let $\underline{w}=\left(w_{m, n}\right)_{m+n \geq 1} \in \mathcal{W}_{1}^{s}$ and $F_{j} \equiv F_{j}\left(H_{f}\right), j=$ $0 \ldots L$, where the functions $F_{j}(r)$ are $C^{s}$ and are bounded together with their derivatives. Write $W:=\sum_{m+n \geq 1} W_{m, n}$ with $W_{m, n}:=W_{m, n}\left[w_{m, n}\right]$. Then

$$
F_{0} W F_{1} W \cdots W F_{L-1} W F_{L}=\widetilde{W}
$$

where $\widetilde{W}:=\widetilde{W}[\underline{\tilde{w}}], \underline{\tilde{w}}:=\left(\widetilde{w}_{M, N}^{(\mathrm{sym})}\right)_{M+N \geq 0}$ with $\widetilde{w}_{M, N}^{(\mathrm{sym})}$ given by the symmetrization $w . r . t$. $k_{(M)}$ and $\tilde{k}_{(N)}$, of the coupling functions

$$
\begin{gathered}
\widetilde{w}_{M, N}\left[r ; k_{(M, N)}\right]=\sum_{\substack{m_{1}+\ldots+m_{L}=M, n_{1}+\ldots+n_{L}=N}} \sum_{\substack{p_{1}, q_{1}, \ldots, p_{L}, q_{L}: \\
m_{\ell}+p_{\ell}+n_{\ell}+q_{\ell} \geq 1}} \prod_{\ell=1}^{L}\left\{\left(\begin{array}{c}
m_{\ell}+p_{\ell} \\
p_{\ell}
\end{array}\right)\left(\begin{array}{c}
n_{\ell}+q_{\ell} \\
q_{\ell}
\end{array}\right)\right\} \\
F_{0}\left[r+\tilde{r}_{0}\right]\langle\Omega| \widetilde{W}_{1}\left[r+r_{1} ; k_{\left(m_{1}, n_{1}\right)}^{(1)}\right] F_{1}\left[H_{f}+r+\tilde{r}_{1}\right] \widetilde{W}_{2}\left[r+r_{2} ; k_{\left(m_{2}, n_{2}\right)}^{(2)}\right] \\
\left.\cdots F_{L-1}\left[H_{f}+r+\tilde{r}_{L-1}\right] \widetilde{W}_{L}\left[r+r_{L} ; k_{\left(m_{L}, n_{L}\right)}^{(L)}\right] \Omega\right\rangle F_{L}\left[r+\tilde{r}_{L}\right],
\end{gathered}
$$

with

$$
\widetilde{W}_{\ell}\left[r ; k_{\left(m_{\ell}, n_{\ell}\right)}\right]:=W_{p_{\ell}, q_{\ell}}^{m_{\ell}, n_{\ell}}\left[\underline{w} \mid r ; k_{\left(m_{\ell}, n_{\ell}\right)}\right] .
$$

For a proof of this theorem see [8, Theorem A.4]. Here we sketch the idea of this proof. Substituting the expansion $W:=\sum_{m+n \geq 1} W_{m, n}$ into (VI.10) we find

$$
\widetilde{W}=\sum_{\substack{m_{1}^{\prime}, n_{1}^{\prime}, \ldots, m_{L}^{\prime}, n_{L}^{\prime} \\ m_{\ell}^{\prime}+n_{\ell}^{\prime} \geq 1}} F_{0} \prod_{i=1}^{L}\left(W_{m_{i}^{\prime}, n_{i}^{\prime}} F_{i}\right) .
$$


Now we want to transform each product on the r.h.s. to the generalized normal form, see Eqn (III.12). Each factor has the creation and annihilation operators entering it explicitly and through the operators $H_{f}$. We do not touch the latter and reshuffle the former.

We pull the annihilation operators, $a$, entering the $W_{m_{i}^{\prime}, n_{i}^{\prime}}$ 's explicitly, to the left and the creation operators, $a^{*}$, to the left. The creation and annihilation operators interchange positions according to the formula

$$
a(k) a^{*}\left(k^{\prime}\right)=a^{*}\left(k^{\prime}\right) a(k)+\delta\left(k-k^{\prime}\right)
$$

Thus they either pass through each other without a change or produce the $\delta$-function (contract with each other). Furthermore, they pass through functions of the photon Hamiltonian operator $H_{f}$ according to the Pull-Through formulae

$$
a(k) F\left[H_{f}\right]=F\left[H_{f}+|k|\right] a(k), F\left[H_{f}\right] a^{*}(k)=a^{*}(k) F\left[H_{f}+|k|\right],
$$

which hold on $\mathcal{H}_{\text {red }}$ in the sense of operator-valued distributions for every bounded and measurable function $F$, see [8, Lemma A.1].

Some of the creation and annihilation operators reach the extreme left and right positions, while the remaining ones contract. The terms with $M$ creation operators reaching the extreme left positions and $N$ annihilation operators reaching the extreme right positions contribute to the $(M, N)$ - formfactor, $\widetilde{w}_{M, N}$, of the operator $\widetilde{W}$.

This is the standard way for proving the Wick theorem on the reduction of operators on Fock spaces to their normal (or Wick) forms, modified by presence of $H_{f}-$ dependent factors. The problem here is that the number of terms generated by various contractions, which is the number of pairs which can be formed by creation and annihilation operators, is, very roughly, of order of $L$ ! for a product of $L$ terms. Therefore a simple majoration of the series for $\widetilde{w}_{M, N}$ will diverge badly. Thus we have to re-sum this series in order to take advantage of possible cancelations. The latter is done by, roughly, representing, for a given $M$ and $N$, the sum over all contractions by a vacuum expectation which effects only the 'contracting' creation and annihilation operators and does not apply to the 'external' ones, i.e. those which reached the extreme positions on the left and right.

As a direct consequence of Theorem VI.1 and Eqns. (IV.7), (IV.9)-(IV.10) and (VI.3), we find a sequence $\underline{\hat{w}}$ such that $H(\underline{\hat{w}})=\mathcal{R}_{\rho}(H(\underline{w}))=S_{\rho}\left(F_{\rho}(H(\underline{w}))\right)$ as follows.

Theorem VI.2. Let $H(\underline{w}) \in \mathcal{D}^{\mu, s}(\rho / 8, \rho / 8, \rho / 8)$. Then $\mathcal{R}_{\rho}(H(\underline{w}))=H(\underline{\hat{w}})$ where $\underline{\hat{w}}=$ $\left(\hat{w}_{M, N}^{(s y m)}\right)_{M+N \geq 0}$ with $\hat{w}_{M, N}^{(\text {sym })}$, the symmetrization w.r.t. $k^{(M)}$ and $\tilde{k}^{(N)}$ (as in Eq. (VI.5)) of 
the kernels

$$
\begin{aligned}
& \hat{w}_{M, N}\left[r ; k_{(M, N)}\right]= \rho^{M+N-1} \sum_{L=1}^{\infty}(-1)^{L-1} \times \\
& \sum_{\substack{m_{1}+\ldots+m_{L}=M, n_{1}+\ldots+n_{L}=N}} \sum_{\substack{p_{1}, q_{1}, \ldots, p_{L}, q_{L}: \\
m_{\ell}+p_{\ell}+n_{\ell}+q_{\ell} \geq \delta_{L}}} \prod_{\ell=1}^{L}\left\{\left(\begin{array}{c}
m_{\ell}+p_{\ell} \\
p_{\ell}
\end{array}\right)\left(\begin{array}{c}
n_{\ell}+q_{\ell} \\
q_{\ell}
\end{array}\right)\right\} V_{\underline{m, p, n, q}}\left[r ; k_{(M, N)}\right],
\end{aligned}
$$

for $M+N \geq 1$, and

$$
\hat{w}_{0,0}[r]=r+\rho^{-1} \sum_{L=2}^{\infty}(-1)^{L-1} \sum_{\substack{p_{1}, q_{1}, \ldots, p_{L}, q_{L}: \\ p_{\ell}+q_{\ell} \geq 1}} \prod_{\ell=1}^{L} V_{\underline{0, p, 0, q}}[r]
$$

for $M=N=0$. Here $\underline{m, p, n, q}:=\left(m_{1}, p_{1}, n_{1}, q_{1}, \ldots, m_{L}, p_{L}, n_{L}, q_{L}\right) \in \mathbb{N}_{0}^{4 L}$, and

$$
\begin{aligned}
& V_{\frac{m, n, q}{2}}\left[r ; k_{(M, N)}\right]:= \\
& \left\langle\Omega, F_{0}\left[H_{f}+r\right] \prod_{\ell=1}^{L}\left\{\widetilde{W}_{\ell}\left[\rho\left(r+r_{\ell}\right) ; \rho k_{\left(m_{\ell}, n_{\ell}\right)}^{(\ell)}\right] F_{\ell}\left[H_{f}+r\right]\right\} \Omega\right\rangle,
\end{aligned}
$$

with $M:=m_{1}+\ldots+m_{L}, N:=n_{1}+\ldots+n_{L}, F_{0}[r]:=\chi_{1}\left[r+\tilde{r}_{0}\right], F_{L}[r]:=\chi_{1}\left[r+\tilde{r}_{L}\right]$ and $F_{\ell}[r]:=\frac{\bar{\chi}_{1}\left[r+\tilde{r}_{\ell}\right]^{2}}{T\left[\rho\left(r+\tilde{r}_{\ell}\right)\right]+E}$, for $\ell=1, \ldots, L-1$. Here the notation introduced in Eqs. (VI.4)(VI.9) and (VI.12) is used.

We remark that Theorem VI.2 determines $\underline{\hat{w}}$ from $\underline{w} \in \mathcal{W}^{\mu, s}$ only as a sequence of integral kernels that define an operator in $\mathcal{B}[\overline{\mathcal{F}}]$. Now we show that $\underline{\hat{w}} \in \mathcal{W}^{\mu, s}$, i.e. $\|\underline{\hat{w}}\|_{\mu, s, \xi}<\infty$. In what follows we use the notation introduced in Eqs. (VI.4)-(VI.9) and (VI.12). To estimate $\underline{\hat{w}}$, we start with the following preparatory lemma

Lemma VI.3. For fixed $L \in \mathbb{N}$ and $\underline{m, p, n, q} \in \mathbb{N}_{0}^{4 L}$, we have $V_{\underline{m, p, n, q}} \in \mathcal{W}_{M, N}^{\mu, s}$ and

$$
\left\|V_{\underline{m, p, n, q}}\right\|_{\mu, s} \leq 4 C_{\chi}^{2} \rho^{\mu} L^{s}\left(\frac{C_{\chi}}{\rho}\right)^{L-1} \prod_{\ell=1}^{L} \frac{\left\|w_{m_{\ell}+p_{\ell}, n_{\ell}+q_{\ell}}\right\|_{\mu, s}}{\sqrt{p_{\ell}^{p_{\ell}} q_{\ell}^{q_{\ell}}}}
$$

with the convention that $p^{p}:=1$ for $p=0$. Here the constant $C_{\chi}$ is given by (IV.12).

This lemma is proven in [2] (Lemma III.10) for the $L^{2}$-version of the norms (III.5) and (III.7) with $s=1$ The extension of this lemma to the norms (III.5) and (III.7) with $s=2$, used in this paper, is straightforward. We present here the proof for $s=0$ and point out how it extends to the $s>0$ case in order to illustrate its simple structure and for references needed later. 
Remark VI.4. The proof of Lemma VI.3 requires taking derivatives of $\chi_{1}[r]$ and $\bar{\chi}_{1}[r]$. Here the main advantage of using the smooth Feshbach map, rather than the (projection) Feshbach map, becomes manifest. If $\chi_{1}[r]$ and $\bar{\chi}_{1}[r]$ were projections, i.e., characteristic functions of intervals, we would inevitably encounter $\delta$-distributions. In fact, the appearance of these $\delta$-distributions are the reason for using (a rather involved mixture of) supremum and $L^{1}$-norms in [7, 8]. In contrast, the proof of Lemma VI.3 is quite straightforward and merely requires summation of geometric series.

Proof. First we note that by the definition of the cut-of function $\chi_{1}(r) \equiv \chi_{r \leq 1}$ (see the paragraph after (III.9) $),\left|F_{i}[r]\right| \leq 1, i=0, L$. Moreover, since $T(r) \geq \frac{7}{8} r, \operatorname{supp} \bar{\chi}_{1} \subset$ $\{r \geq 1\}$ and $|E| \leq \frac{1}{8} \rho$, we have that, for $\ell=1, \ldots, L-1$,

$$
\left|F_{\ell}[r]\right| \leq\left|\frac{\bar{\chi}_{1}^{2}\left[r+\tilde{r}_{\ell}\right]}{T\left[\rho\left(r+\tilde{r}_{\ell}\right)\right]-E}\right| \leq \frac{4}{3 \rho} .
$$

Now, we estimate $\left|V_{\underline{m, p, n, q}}\right|$, using that $|\langle\Omega, A \Omega\rangle| \leq\|A\|_{\text {op }}$, for any $A \in \mathcal{B}\left[\mathcal{H}_{\text {red }}\right]$. We have that

$$
\begin{aligned}
& \left|V_{\underline{m, p, n, q}}\left[r ; k_{(M, N)}\right]\right| \\
& \quad \leq \prod_{\ell=0}^{L}\left\|F_{\ell}\left[H_{f}+r\right]\right\|_{\mathrm{op}} \prod_{\ell=1}^{L}\left\|\widetilde{W}_{\ell}\left[\rho\left(r+r_{\ell}\right) ; \rho k_{\left(m_{\ell}, n_{\ell}\right)}^{(\ell)}\right]\right\|_{\mathrm{op}} .
\end{aligned}
$$

Using (III.17) and letting $\ell_{j}$ to be defined by the property that the vector $k_{\left(m_{\ell_{j}}, n_{\ell_{j}}\right)}^{\left(\ell_{\ell}\right)}$ contains $k_{j}$ among its 3 -dimensional components, we arrive at

$$
\begin{aligned}
& \left\|V_{\underline{m, p, n, q}}\right\|_{\mu}=\left.\max _{j} \sup _{r \in I, k_{(M, N)} \in B_{1}^{M+N}}|| k_{j}\right|^{-\mu} V_{\underline{m, p, n, q}}\left[r ; k_{(M, N)}\right] \mid \\
& \leq\left(\frac{4}{3 \rho}\right)^{L-1} \max _{j} \prod_{\ell \neq \ell_{j}}^{1, L}\left\{\sup _{r \in I, k_{\left(m_{\ell}, n_{\ell}\right)}^{(\ell)} \in B_{1}^{m_{\ell}+n_{\ell}}}\left\|\widetilde{W}_{\ell}\left[\rho r ; \rho k_{\left(m_{\ell}, n_{\ell}\right)}^{(\ell)}\right]\right\|_{\mathrm{op}}\right\} \\
& \sup _{r \in I, k_{\left(m_{\ell_{j}}, n_{\ell_{j}}\right)}^{\left(\ell_{j}\right)} \in B_{1}^{m_{\ell_{j}}+n_{\ell_{j}}}}\left|k_{j}\right|^{-\mu}\left\|\widetilde{W}_{\ell_{j}}\left[\rho r ; \rho k_{\left(m_{\ell_{j}}, n_{\ell_{j}}\right)}^{\left(\ell_{j}\right)}\right]\right\|_{\text {op }} \\
& \leq\left(\frac{4}{3 \rho}\right)^{L-1} \rho^{\mu} \max _{j} \prod_{\ell \neq \ell_{j}}^{1, L}\left\{\sup _{r \in I, k_{\left(m_{\ell}, n_{\ell}\right)}^{(\ell)} \in B_{1}^{m_{\ell}+n_{\ell}}}\left\|\widetilde{W}_{\ell}\left[r ; k_{\left(m_{\ell}, n_{\ell}\right)}^{(\ell)}\right]\right\|_{\mathrm{op}}\right\}
\end{aligned}
$$




$$
\sup _{r \in I, k_{\left(m_{\ell_{j}}, n_{\ell_{j}}\right)}^{\left(\ell_{j}\right)} \in B_{1}^{m_{\ell_{j}}+n_{\ell_{j}}}}\left|k_{j}\right|^{-\mu}\left\|\widetilde{W}_{\ell_{j}}\left[r ; k_{\left(m_{\ell_{j}}, n_{\ell_{j}}\right)}^{\left(\ell_{j}\right)}\right]\right\|_{\mathrm{op}} .
$$

We now convert the operator norms on the right side of (VI.20) into the coupling functions norms. To this end we use, pointwise in $k_{\left(m_{\ell}, n_{\ell}\right)}^{(\ell)}$ a.e., inequality (III.11) in Theorem III.1 to obtain for any $\mu \geq 0$

$$
\begin{aligned}
& \max _{j} \sup _{r \in I, k_{\left(m_{\ell}, n_{\ell}\right)}^{(\ell)} \in B_{1}^{m_{\ell}+n_{\ell}}}\left|k_{j}\right|^{-\mu}\left\|\widetilde{W}_{\ell}\left[r ; k_{\left(m_{\ell}, n_{\ell}\right)}^{(\ell)}\right]\right\|_{\mathrm{op}} \\
& \leq \frac{1}{\sqrt{p_{p_{\ell}}^{(\ell)} q_{q_{\ell}}^{(\ell)}}} \max _{j} \sup _{r \in I, k_{\left(m_{\ell}, n_{\ell}\right.}^{(\ell)} \in B_{1}^{m_{\ell}+n_{\ell}}}\left|k_{j}\right|^{-\mu}\left\|w_{m_{\ell}+p_{\ell}, n_{\ell}+q_{\ell}}\left[\cdot ; k_{\left(m_{\ell}\right)}^{(\ell)}, \cdot ; \tilde{k}_{\left(n_{\ell}\right)}^{(\ell)}, \cdot\right]\right\|_{0} \\
& \leq \frac{1}{\sqrt{p_{p_{\ell}}^{(\ell)} q_{q_{\ell}}^{(\ell)}}}\left\|w_{m_{\ell}+p_{\ell}, n_{\ell}+q_{\ell}}\right\|_{\mu} .
\end{aligned}
$$

This estimate with $\mu=0$ if $\ell \neq \ell_{j}$ and $\mu \geq 0$ if $\ell=\ell_{j}$, inserted into the $\ell^{\text {th }}$ factor on the right side of (VI.20), yields (VI.17) with $s=0$.

To estimate the norm $\left\|V_{m, p, n, q}\right\|_{\mu, s}$ with $s=1,2$ we need the bounds

$$
\left|\partial_{r}^{s} F_{\ell}[r]\right| \leq \frac{C_{\chi}}{\rho}
$$

where the constant $C_{\chi}$ is given in (IV.12). These bounds are obtained similarly to (VI.18), using the inequality

$$
\begin{aligned}
\left|\partial_{r} F_{\ell}[r]\right| & \leq\left|\frac{2 \bar{\chi}_{1}\left[r+\tilde{r}_{\ell}\right] \partial_{r} \bar{\chi}_{1}\left[r+\tilde{r}_{\ell}\right]}{T\left[\rho\left(r+\tilde{r}_{\ell}\right)\right]-E}\right| \\
& +\left|\frac{\bar{\chi}_{1}^{2}\left[r+\tilde{r}_{\ell}\right] \rho \partial_{r} T\left[z ; \rho\left(r+\tilde{r}_{\ell}\right)\right]}{\left(T\left[\rho\left(r+\tilde{r}_{\ell}\right)\right]-E\right)^{2}}\right|
\end{aligned}
$$

and a similar inequality for $\left|\partial_{r}^{2} F_{\ell}[r]\right|$.

To estimate $\left\|V_{m, p, n, q}\right\|_{\mu, s}$ with $s=1,2$ we apply the operator $\partial_{r}^{n}$ to (VI.16) and use the Leibnitz rule of differentiation of products $s$ times to obtain (VI.17).

We are now prepared to prove the estimates in Theorem IV.3. Recall that we assume $\rho \leq 1 / 2$ and we choose $\xi=1 / 4$. First, we apply Lemma VI.3 to (VI.14) and use that 
$\left(\begin{array}{c}m+p \\ p\end{array}\right) \leq 2^{m+p}$. This yields

$$
\begin{aligned}
& \left\|\hat{w}_{M, N}\right\|_{\mu, s} \leq \sum_{L=1}^{\infty} 4 C_{\chi} \rho^{\mu} L^{s}\left(\frac{C_{\chi}}{\rho}\right)^{L}(2 \rho)^{M+N} \\
& \sum_{\substack{m_{1}+\ldots+m_{L}=M, n_{1}+\ldots+n_{L}=N}} \sum_{\substack{p_{1}, q_{1}, \ldots, p_{L}, q_{L}: \\
m_{\ell}+p_{\ell}+n_{\ell}+q_{\ell} \geq 1}} \prod_{\ell=1}^{L}\left\{\left(\frac{2}{\sqrt{p_{\ell}}}\right)^{p_{\ell}}\left(\frac{2}{\sqrt{q_{\ell}}}\right)^{q \ell}\left\|w_{m_{\ell}+p_{\ell}, n_{\ell}+q_{\ell}}\right\|_{\mu, s}\right\} .
\end{aligned}
$$

Using the definition (III.17), the inequality $2 \rho \leq 1$, we derive the following bound for $\underline{\hat{w}}_{1}:=\left(\hat{w}_{M, N}\right)_{M+N \geq 1}$,

$$
\begin{aligned}
&\left\|\underline{\hat{w}}_{1}\right\|_{\mu, s, \xi}:=\sum_{M+N \geq 1} \xi^{-(M+N)}\left\|\hat{w}_{M, N}\right\|_{\mu, s} \\
& \leq \quad 8 C_{\chi} \rho^{1+\mu} \sum_{L=1}^{\infty} L^{s}\left(\frac{C_{\chi}}{\rho}\right)^{L} \sum_{M+N \geq 1} \sum_{\substack{m_{1}+\ldots+m_{L}=M, n_{1}+\ldots+n_{L}=N}} \sum_{\substack{p_{1}, q_{1}, \ldots, p_{L}, q_{L}: \\
m_{\ell}+p_{\ell}+n_{\ell}+q_{\ell} \geq 1}} \\
& \prod_{\ell=1}^{L}\left\{\left(\frac{2 \xi}{\sqrt{p_{\ell}}}\right)^{p_{\ell}}\left(\frac{2 \xi}{\sqrt{q_{\ell}}}\right)^{q_{\ell}} \xi^{-\left(m_{\ell}+p_{\ell}+n_{\ell}+q_{\ell}\right)}\left\|w_{m_{\ell}+p_{\ell}, n_{\ell}+q_{\ell}}\right\|_{\mu, s}\right\} \\
& \leq 8 C_{\chi} \rho^{1+\mu} \sum_{L=1}^{\infty} L^{s}\left(\frac{C_{\chi}}{\rho}\right)^{L} \\
&\left\{\sum_{m+n \geq 1}\left(\sum_{p=0}^{m}\left(\frac{2 \xi}{\sqrt{p}}\right)^{p}\right)\left(\sum_{q=0}^{n}\left(\frac{2 \xi}{\sqrt{q}}\right)^{q}\right) \xi^{-(m+n)}\left\|w_{m, n}\right\|_{\mu, s}\right\}^{L} .
\end{aligned}
$$

Using the assumption $\xi=1 / 4$ and the estimate $\sum_{p=0}^{m}(2 \xi / \sqrt{p})^{p} \leq \sum_{p=0}^{\infty}(2 \xi)^{p}=\frac{1}{1-2 \xi}$, and recalling the definitions $\underline{w}_{1}:=\left(w_{m, n}\right)_{m+n \geq 1}$ and $\left\|\underline{w}_{1}\right\|_{\mu, s, \xi}:=$ $\sum_{M+N \geq 1} \xi^{-(m+n)}\left\|w_{m, n}\right\|_{\mu, s}$, we obtain

$$
\left\|\underline{\hat{w}}_{1}\right\|_{\mu, s, \xi} \leq 8 C_{\chi} \rho^{\mu+1} \sum_{L=1}^{\infty} L^{s} B^{L}
$$

where

$$
B:=\frac{C_{\chi}}{\rho(1-2 \xi)^{2}}\left\|\underline{w}_{1}\right\|_{\mu, s, \xi} .
$$

Note that in (VI.23) we have dropped the factor $p^{-p / 2}$ gained in Theorem III.1. Our assumption, $\gamma \leq\left(8 C_{\chi}\right)^{-1} \rho$, also insures that

$$
B \leq \frac{4 C_{\chi} \gamma}{\rho} \leq \frac{1}{2}
$$


Thus the geometric series in the last line of (VI.23) is convergent. We obtain for $s=0,1,2$

$$
\sum_{L=1}^{\infty} L^{s} B^{L} \leq 8 B
$$

Inserting (VI.26) into (VI.23), we see that the r.h.s. of (VI.23) is bounded by $64 C_{\chi} \rho^{1+\mu} B$ which, remembering the definition of $B$ gives

$$
\left\|\underline{\hat{w}}_{1}\right\|_{\mu, s, \xi} \leq 256 C_{\chi}^{2} \rho^{\mu}\left\|\underline{w}_{1}\right\|_{\mu, s, \xi} .
$$

Next, we estimate $\hat{w}_{0,0}$. We analyze the expression (VI.15). Using estimate Eq. (VI.17) with $\underline{m}=0, \underline{n}=0$ (and consequently, $M=0, N=0$ ), we find

$$
\rho^{-1}\left\|V_{\underline{0, p, 0, q}}\right\|_{\mu, s} \leq 2 L^{s} C_{\chi}^{L+1} \rho^{-L} \prod_{\ell=1}^{L} \frac{\left\|w_{p_{\ell}, q_{\ell}}\right\|_{\mu, s}}{\sqrt{p_{\ell}^{p_{\ell}} q_{\ell}^{q_{\ell}}}} .
$$

In fact, examining the proof of Lemma VI.3 more carefully we see that the following, slightly stronger estimate is true

$$
\rho^{-1}\left\|\partial_{r}^{s} V_{0, p, 0, q}\right\|_{\mu, 0} \leq 2 L^{s} C_{\chi}^{L+1} \rho^{-L+s} \prod_{\ell=1}^{L} \frac{\left\|w_{p_{\ell}, q_{\ell}}\right\|_{\mu, s}}{\sqrt{p_{\ell}^{p_{\ell}} q_{\ell}^{q_{\ell}}}} .
$$

Now, using (VI.29) and $\sum_{p+q \geq 1}\left\|w_{p, q}\right\|_{\mu, s} \leq \xi \sum_{p+q \geq 1} \xi^{-p-q}\left\|w_{p, q}\right\|_{\mu, s}=:\left\|\underline{w}_{1}\right\|_{\mu, s, \xi}$, where, recall, $\underline{w}_{1}:=\left(w_{m, n}\right)_{m+n \geq 1}$, we obtain

$$
\begin{aligned}
& \rho^{-1} \sum_{L=2}^{\infty} \sum_{\substack{p_{1}, q_{1}, \ldots, p_{L}, q_{L}: \\
p_{\ell}+q_{\ell} \geq 1}} \sup _{r \in I}\left|\partial_{r}^{s} V_{\underline{0, p, 0, q}}[r]\right| \\
\leq & 2 C_{\chi} \rho^{s} \sum_{L=2}^{\infty} L^{s}\left(\frac{C_{\chi}}{\rho}\right)^{L}\left\{\sum_{p+q \geq 1}\left\|w_{p, q}\right\|_{\mu, s}\right\}^{L} \\
\leq & 2 C_{\chi} \rho^{s} \sum_{L=2}^{\infty} L^{s} D^{L},
\end{aligned}
$$

where $D:=C_{\chi} \rho^{-1} \xi\left\|_{w_{1}}\right\|_{\mu, s, \xi}$. Now, since $D \leq C_{\chi} \xi \rho^{-1} \gamma \leq \xi / 8=1 / 16$, we have, similarly to (VI.26), that $\sum_{L=2}^{\infty} L^{s} D^{L} \leq 12 D^{2}$ for $s=0,1,2$. Hence we find

$$
\begin{aligned}
& \rho^{-1} \sum_{L=2}^{\infty} \sum_{\substack{p_{1}, q_{1}, \ldots, p_{L}, q_{L}: \\
p_{\ell}+q_{\ell} \geq 1}} \sup _{r \in I}\left|\partial_{r}^{s} V_{\underline{0, p, 0, q}}[r]\right| \\
& \leq \quad 24 C_{\chi} \rho^{s}\left(\frac{C_{\chi} \xi}{\rho}\left\|\underline{w}_{1}\right\|_{\mu, s, \xi}\right)^{2},
\end{aligned}
$$


for $s=0,1,2$.

We set $\widehat{E}:=\hat{w}_{0,0}[0]$. Since $E=w_{0,0}[0]$, Eqs. (VI.15) and (VI.31) yield

$$
\left|\widehat{E}-\rho^{-1} E\right| \leq 24 C_{\chi}\left(\frac{C_{\chi} \xi}{\rho}\left\|\underline{w}_{1}\right\|_{\mu, 0, \xi}\right)^{2}
$$

Next, writing $\widehat{T}[r]:=\hat{w}_{0,0}[r]-\hat{w}_{0,0}[0]$, we find furthermore that

$$
\begin{aligned}
& \sup _{r \in[0, \infty)}\left|\widehat{T}^{\prime}[r]-1\right|=\sup _{r \in[0, \infty)}\left|\partial_{r} \hat{w}_{0,0}[r]-1\right| \\
& \leq \sup _{r \in[0, \infty)}\left|T^{\prime}[r]-1\right|+24 C_{\chi} \rho\left(\frac{C_{\chi} \xi}{\rho}\left\|\underline{w}_{1}\right\|_{\mu, 1, \xi}\right)^{2} .
\end{aligned}
$$

Now, recall that $\left|T^{\prime}[r]-1\right| \leq \beta$ and $\left\|\underline{w}_{1}\right\|_{\mu, s, \xi} \leq \gamma$. Hence Eqns (VI.32), (VI.33), and (VI.27) give (IV.13) with $\alpha^{\prime}=24 C_{\chi}\left(\frac{C_{\chi} \xi \gamma}{\rho}\right)^{2}, \beta^{\prime}=\beta+24 C_{\chi}\left(\frac{C_{\chi} \xi \gamma}{\rho}\right)^{2}$ and $\gamma^{\prime}=$ $256 C_{\chi}^{2} \rho^{\mu} \gamma$. Remembering that $\xi=\sqrt{\rho} /\left(4 C_{\chi}\right)$ we conclude that the statement of Theorem IV.3 holds.

Remark VI.5. In the proof the limiting absorption principle (LAP) in [17] to estimate $\left\|V_{\underline{m, p, n, q}}\right\|_{\mu, s}$, with $s=1,2$, (see Lemma VI.3) instead of the operator $\partial_{r}^{n}$, we apply the operator $\partial_{r}^{n}\left(k \partial_{k}\right)^{q}$ to (VI.16). Here $q:=\left(q_{1}, \ldots, q_{M+N}\right),\left(k \partial_{k}\right)^{q}:=\prod_{1}^{M+N}\left(k_{j} \cdot \nabla_{k_{j}}\right)^{q_{j}}$, with $k_{m+j}:=\tilde{k}_{j}$, and the indices $n$ and $q$ satisfy $0 \leq n+|q| \leq s$.

Remark VI.6. For the proof of the limiting absorption principle in [17] we also need the following estimate (here we use that $\widehat{T}^{\prime \prime}[r]=\partial_{r}^{2} \hat{w}_{0,0}[r]$ )

$$
\sup _{r \in[0, \infty)}\left|\widehat{T}^{\prime \prime}[r]\right| \leq \rho \sup _{r \in[0, \infty)}\left|T^{\prime \prime}[r]\right|+24 C_{\chi} \rho^{2}\left(\frac{C_{\chi} \xi}{\rho}\left\|\underline{w}_{1}\right\|_{\mu, 2, \xi}\right)^{2} .
$$

\section{Appendix II: Construction of Eigenvalues and Eigen- vectors}

In this appendix we prove that the value $E:=e\left(H_{s}\right)+H_{u}$ we constructed in Section $\mathrm{V}$ is the ground state energy of the Hamiltonian $H$ under consideration (see Theorem V.3) and we construct the corresponding the ground state energy. We use the definitions of Section V.

Theorem VII.1. Let $H \in \mathcal{D}$ Then the value $E:=e\left(H_{s}\right)+H_{u}$ where $e\left(H_{s}\right)$ is given in Theorem V.1, is a simple eigenvalue of the operator $H$. The corresponding eigenfunction is given constructively in Eq. (VII.13) below. 
Proof. Let $H^{(0)}:=H-E \in \mathcal{M}_{s}$. We define a sequence of operators $\left(H^{(n)}\right)_{n=0}^{\infty}$ in $\mathcal{W}_{o p}^{\mu, s} \subseteq \mathcal{B}\left(\mathcal{H}_{\text {red }}\right)$ by $H^{(n)}:=\mathcal{R}_{\rho}^{n}\left(H^{(0)}\right)$. We will also need the following representation for $S_{\rho}$ :

$$
S_{\rho}(A)=: \Gamma_{\rho} A \Gamma_{\rho}^{*},
$$

where $\Gamma_{\rho}$ is the unitary dilatation on $\mathcal{F}$ defined by this formula and $\Gamma_{\rho} \Omega=\Omega$. Then the definition (IV.13) of $\mathcal{R}_{\rho}$ implies that, for all integers $n \geq 0$,

$$
H^{(n)}=\frac{1}{\rho} \Gamma_{\rho}\left(F_{\rho}\left(H^{(n-1)}\right)\right) \Gamma_{\rho}^{*}
$$

where, recall, $F_{\rho}:=F_{\tau \chi_{\rho}}$ with $\tau(H):=W_{0,0}$ (see Eqn (IV.1)). We will use the operators $Q_{\tau \chi}$ defined in (II.6). It is easy to show (see [2]) that these operators satisfy the identity $H Q_{\tau \chi}=\chi F_{\tau \chi}(H)$. Let

$$
Q^{(n)}:=Q_{\tau \chi_{\rho}}\left(H^{(n)}\right),
$$

Then the equation $H^{(n)} Q^{(n)}=\chi_{\rho} F_{\rho}\left(H^{(n)}\right)$ together with (VII.2), implies the intertwining property

$$
H^{(n-1)} Q^{(n-1)} \Gamma_{\rho}^{*}=\rho \Gamma_{\rho}^{*} \chi_{1} H^{(n)} .
$$

Eq. (VII.4) is the key identity for the proof of the existence of an eigenvector with the eigenvalue $e$.

For the construction of this eigenvector, for non-negative integers $\beta$ we define vectors $\Psi_{k}$ in $\mathcal{H}_{\text {red }}$ by setting $\Psi_{0}:=\Omega$ and

$$
\Psi_{k}:=Q^{(0)} \Gamma_{\rho}^{*} Q^{(1)} \Gamma_{\rho}^{*} \cdots Q^{(k-1)} \Omega
$$

We first show that this sequence is convergent, as $k \rightarrow \infty$. To this end, we observe that $\Omega=\Gamma_{\rho}^{*} \chi_{\rho} \Omega$ and hence

$$
\Psi_{k+1}-\Psi_{k}=Q^{(0)} \Gamma_{\rho}^{*} Q^{(1)} \Gamma_{\rho}^{*} \cdots Q^{(k-1)} \Gamma_{\rho}^{*}\left(Q^{(k)}-\chi_{\rho}\right) \Omega
$$

Since $\left\|\chi_{\rho}\right\| \leq 1$, this implies that

$$
\left\|\Psi_{k+1}-\Psi_{k}\right\| \leq\left\|Q^{(k)}-\chi_{\rho}\right\|_{\text {op }} \prod_{j=0}^{\beta-1}\left\{1+\left\|Q^{(j)}-\chi_{\rho}\right\|_{\mathrm{op}}\right\} .
$$

To estimate the terms on the r.h.s. we consider the $j$-th step Hamiltonian $H^{(j)}$. As in the proof of proposition VI.5 we write $H^{(j)}$ as

$$
H^{(j)}=E_{j} \cdot \mathbf{1}+T_{j}+W_{j}
$$


with

$$
\left|E_{j}\right| \leq 8 \alpha_{j} \text { and }\left\|W_{j}\right\|_{\text {op }} \leq \gamma_{j} \leq \frac{\rho}{16} .
$$

Recalling the definition (II.6) of $Q_{(j)}$, we have

$$
\begin{aligned}
& \chi_{\rho}-Q_{(j)}= \\
& \bar{\chi}_{\rho}\left(E_{j}+T_{j}+\bar{\chi}_{\rho} W_{j} \bar{\chi}_{\rho}\right)^{-1} \bar{\chi}_{\rho} W_{j} \chi_{\rho} .
\end{aligned}
$$

By (VII.9), for all $j \in \mathbb{N}$, we may estimate

$$
\left\|\chi_{\rho}-Q_{(j)}\right\|_{\text {op }} \leq\left(\frac{\rho}{8}-\left\|W_{j}\right\|_{\text {op }}\right)^{-1}\left\|W_{j}\right\|_{\text {op }} \leq \frac{16 \gamma_{j}}{\rho} .
$$

Inserting this estimate into (VII.7) and using that $\prod_{j=0}^{\infty}\left(1+\lambda_{j}\right) \leq \exp \left[\sum_{j=0}^{\infty} \lambda_{j}\right]$, for $\lambda_{j} \geq 0$, we obtain

$$
\begin{aligned}
\left\|\Psi_{k+1}-\Psi_{k}\right\| & \leq \frac{16 \gamma_{k}}{\rho} \prod_{j=0}^{k-1}\left\{1+\frac{16 \gamma_{j}}{\rho}\right\} \\
& \leq \frac{16 \gamma_{k}}{\rho} \exp \left[32 \gamma_{0} \rho^{-1}\right]
\end{aligned}
$$

where we have used that $\sum_{j=0}^{\infty} \gamma_{j} \leq 2 \gamma_{0}$ (recall the definition of $\gamma_{j}$ after Eqn (V.1). Since $\sum_{j=0}^{\infty} \gamma_{j}<\infty$, we see that the sequence $\left(\Psi_{k}\right)_{k \in \mathbb{N}_{0}}$ of vectors in $\mathcal{H}_{\text {red }}$ is convergent, and its limit

$$
\Psi_{\infty}:=\lim _{k \rightarrow \infty} \Psi_{k},
$$

satisfies the estimate

$$
\left\|\Psi_{\infty}-\Omega\right\|=\left\|\Psi_{\infty}-\Psi_{0}\right\| \leq \frac{32 \gamma_{0}}{\rho} \exp \left[32 \gamma_{0} \rho^{-1}\right]
$$

which guarantee that $\Psi_{(\infty)} \neq 0$.

The vector $\Psi_{\infty}$ constructed above is an element of the kernel of $H^{(0)}$, as we will now demonstrate. Observe that, thanks to (VII.4),

$$
\begin{aligned}
H^{(0)} \Psi_{k} & =\left(H^{(0)} Q^{(0)} \Gamma_{\rho}^{*}\right)\left(Q^{(1)} \Gamma_{\rho}^{*} \cdots Q^{(k-1)} \Omega\right) \\
& =\rho \Gamma_{\rho}^{*} \chi_{1}\left(H^{(1)} Q^{(1)} \Gamma_{\rho}^{*}\right)\left(Q^{(2)} \Gamma_{\rho}^{*} \cdots Q^{(k-1)} \Omega\right) \\
& \vdots \\
& =\rho^{k}\left(\Gamma_{\rho}^{*} \chi_{1}\right)^{k} H^{(k)} \Omega .
\end{aligned}
$$


Eq (VII.8) together with the estimate (VII.9) and the relation $T_{k} \Omega=0$ implies that

$$
\begin{aligned}
\left\|H^{(k)} \Omega\right\| & =\left\|\left(W_{k}+E_{k}\right) \Omega\right\| \\
& \leq \gamma_{k}+8 \alpha_{k}^{2} \leq 2 \gamma_{k} .
\end{aligned}
$$

Summarizing VII.15) VII.16) and using that the operator norm of $\Gamma_{\rho}^{*} \chi_{1}$ is bounded by 1 , we arrive at

$$
\left\|H^{(0)} \Psi_{k}\right\| \leq 2 \gamma_{k} \rightarrow 0
$$

as $k \rightarrow \infty$. Since $H^{(0)} \in \mathcal{B}\left(\mathcal{H}_{\text {red }}\right)$ is continuous, (VII.17) implies that

$$
H^{(0)} \Psi_{\infty}=\lim _{k \rightarrow \infty}\left(H^{(0)} \Psi_{k}\right)=0
$$

Thus 0 is an eigenvalue of the operator $H^{(0)}:=H-E$, i.e. $E$ is an eigenvalue of the operator $H$, with the eigenfunction $\Psi_{\infty}$.

\section{Appendix III: Analyticity of all Parts of $H(\underline{w})$}

Let $S$ be an open set in a Banach space $\mathcal{B}$. Below the analyticity is understood in the sense described in the paragraph preceding Theorem V.1.

Proposition VIII.1 ([[19]). Suppose that $\lambda \mapsto H\left(\underline{w}^{\lambda}\right)$ is analytic in $\lambda \in S$ and that $H\left(\underline{w}^{\lambda}\right)$ belongs to some polydisc $\mathcal{D}(\alpha, \beta, \gamma)$ for all $\lambda \in S$. Then:

$$
\lambda \mapsto w_{0,0}^{\lambda}\left(H_{f}\right) \quad \text { and } \quad \lambda \mapsto W\left(\underline{w}^{\lambda}\right)
$$

are analytic in $\lambda \in S$.

Proof. Recall that $B_{1}=\left\{k \in \mathbb{R}^{3}:|k| \leq 1\right\}$ and that an operator $A$ is called $H_{f}$-bounded iff the operator $A\left(H_{f}+1\right)^{-1}$ is bounded. Let $P_{1}$ denote the projection onto the one boson subspace of $\mathcal{F}$, which is isomorphic to $L^{2}\left(\mathbb{R}^{3}\right)$. Then $P_{1} H\left(\underline{w}^{\lambda}\right) P_{1}$, like $H\left(\underline{w}^{\lambda}\right)$, is analytic. We write

$$
\begin{aligned}
P_{1} H\left(\underline{w}^{\lambda}\right)\left(H_{f}+1\right)^{-1} P_{1} & =P_{1} w_{0,0}^{\lambda}\left(H_{f}\right)\left(H_{f}+1\right)^{-1} P_{1}+P_{1} W_{1,1}\left(\underline{w}^{\lambda}\right)\left(H_{f}+1\right)^{-1} P_{1} \\
& =D_{\lambda}+K_{\lambda},
\end{aligned}
$$

where $D_{\lambda}$ denotes multiplication with $w_{0,0}^{\lambda}(\omega)(\omega+1)^{-1}, \omega:=|k|$, and $K_{\lambda}$ is the Hilbert Schmidt operator with kernel

$$
M_{\lambda}(k, \tilde{k})=w_{1,1}^{\lambda}(0, k, \tilde{k})(\tilde{\omega}+1)^{-1}
$$


whose support belongs to $B_{1} \times B_{1}$. In what follows if an operator family has a factor $\left(H_{f}+1\right)^{-1}$ standing on its right, then the analyticity is understood in then operator norm.

Our strategy is to show first that $K_{\lambda}$ and hence

$$
P_{1} w_{0,0}^{\lambda}\left(H_{f}\right)\left(H_{f}+1\right)^{-1} P_{1}=P_{1} H\left(w^{\lambda}\right)\left(H_{f}+1\right)^{-1} P_{1}-K_{\lambda}
$$

are analytic. Then we show that $\lambda \mapsto w_{0,0}^{\lambda}\left(H_{f}\right)$ is analytic. The analyticity of $\lambda \mapsto$ $W\left(\underline{w}^{\lambda}\right)=H\left(\underline{w}^{\lambda}\right)-w_{0,0}^{\lambda}\left(H_{f}\right)$ then follows.

Step 1: $K_{\lambda}$ is analytic.

For each $n \in \mathbb{N}$ let $\left\{Q_{i}^{(n)}\right\}_{i}$ be a collection of $n$ measurable subsets of $B_{1}$ such that

$$
B_{1}=\bigcup_{i=1}^{n} Q_{i}^{(n)}, \quad Q_{i}^{(n)} \cap Q_{j}^{(n)}=\emptyset, \quad i \neq j
$$

and

$$
\left|Q_{i}^{(n)}\right| \leq \frac{\text { const }}{n}
$$

Let $\chi_{i}^{(n)}$ denote the operator on $L^{2}\left(B_{1}\right)$ of multiplication with $\chi_{Q_{i}^{(n)}}$. Then for $i \neq j$, $\chi_{i}^{(n)} D_{\lambda} \chi_{j}^{(n)}=0$ because $\chi_{i}^{(n)}$ and $\chi_{j}^{(n)}$ have disjoint support and commute with $D_{\lambda}$. Together with (VIII.1) this implies that

$$
\chi_{i}^{(n)} K_{\lambda} \chi_{j}^{(n)}=\chi_{i}^{(n)} P_{1} H\left(\underline{w}^{\lambda}\right)\left(H_{f}+1\right)^{-1} P_{1} \chi_{j}^{(n)}, \quad \text { for } i \neq j .
$$

Since the right hand side is analytic, so is the left hand side and hence

$$
K_{\lambda}^{(n)}=\sum_{i \neq j} \chi_{i}^{(n)} K_{\lambda} \chi_{j}^{(n)}
$$

is analytic. It follows that $\lambda \mapsto\left\langle\varphi, K_{\lambda}^{(n)} \psi\right\rangle$ is analytic for all $\varphi, \psi$ in $L^{2}\left(B_{1}\right)$. Now let $\varphi, \psi \in C\left(B_{1}\right)$. Then

$$
\begin{aligned}
& \left|\left\langle\varphi, K_{\lambda}^{(n)} \psi\right\rangle-\left\langle\varphi, K_{\lambda} \psi\right\rangle\right| \\
& =\left|\int_{B_{1} \times B_{1}} \bar{\varphi}(x) \psi(y) M_{\lambda}(x, y) \sum_{i=1}^{n} \chi_{i}^{(n)}(x) \chi_{i}^{(n)}(y) d x d y\right| \\
& \leq\|\varphi\|_{\infty}\|\psi\|_{\infty}\left\|K_{\lambda}\right\|_{\mathrm{HS}}\left(\sum_{i=1}^{n}\left|Q_{i}^{(n)}\right|^{2}\right)^{1 / 2} \longrightarrow 0, \quad(n \rightarrow \infty),
\end{aligned}
$$


uniformly in $\lambda$, because the Hilbert Schmidt norm $\left\|K_{\lambda}\right\|_{\mathrm{HS}}$ is bounded uniformly in $\lambda$ (in fact, it is bounded by $\gamma)$. This proves that $\left\langle\varphi, K_{\lambda} \psi\right\rangle$ is analytic for all $\varphi, \psi \in C\left(B_{1}\right)$. Since $C\left(B_{1}\right)$ is dense in $L^{2}\left(B_{1}\right)$, an other approximate argument using $\sup _{\lambda}\left\|K_{\lambda}\right\|<\infty$ shows that $\left\langle\varphi, K_{\lambda} \psi\right\rangle$ is analytic for all $\varphi, \psi \in L^{2}\left(B_{1}\right)$. Therefore $\lambda \mapsto K_{\lambda}$ is analytic [22].

Step 2: For each $k \in \mathbb{R}^{3}, w_{0,0}^{\lambda}(|k|)(\omega+1)^{-1}$ is an analytic function of $\lambda$.

For each $n \in \mathbb{N}$ let $f_{k, n} \in L^{2}\left(B_{1}\right)$ denote a multiple of the characteristic function of $B_{1 / n}(k)$ with $\left\|f_{k, n}\right\|=1$. By the continuity of $w_{0,0}^{\lambda}(|k|)$ as a function of $k$

$$
\begin{aligned}
w_{0,0}^{\lambda}(|k|)(\omega+1)^{-1} & =\lim _{n \rightarrow \infty} \int_{\mathbb{R}^{3}}\left|f_{k, n}(x)\right|^{2} w_{0,0}^{\lambda}(|x|)(|x|+1)^{-1} d x \\
& =\lim _{n \rightarrow \infty}\left\langle a^{*}\left(f_{k, n}\right) \Omega, w_{0,0}^{\lambda}\left(H_{f}\right)\left(H_{f}+1\right)^{-1} a^{*}\left(f_{k, n}\right) \Omega\right\rangle .
\end{aligned}
$$

Since $a^{*}\left(f_{k, n}\right) \Omega \in P_{1} \mathcal{F}$ the expression $\langle\cdots\rangle$, before taking the limit, is an analytic function of $\lambda$. By assumption on $w_{0,0}^{\lambda}$, this function is Lipschitz continuous with respect to $|k|$ uniformly in $\lambda$. Therefore the convergence in (VIII.4) is uniform in $\lambda$ and hence $w_{0,0}^{\lambda}(|k|)(\omega+1)^{-1}$ is analytic by the Weierstrass approximation theorem from complex analysis.

Step 3: $w_{0,0}^{\lambda}\left(H_{f}\right)$ is analytic.

By the spectral theorem

$$
\left\langle\varphi, w_{0,0}^{\lambda}\left(H_{f}\right)\left(H_{f}+1\right)^{-1} \varphi\right\rangle=\int_{[0, \infty)} w_{0,0}^{\lambda}(x)(x+1)^{-1} d \mu_{\varphi}(x) .
$$

By an application of Lebesgue's dominated convergence theorem, using $\sup _{\lambda} \| w_{0,0}^{\lambda}(x+$ $1)^{-1} \|<\infty$, we see that the right hand side, which we call $\varphi(\lambda)$, is a continuous function of $\lambda$. Therefore

$$
\int_{\Gamma} \varphi(\lambda) d \lambda=\int_{[0,1]}\left(\int_{\Gamma} w_{0,0}^{\lambda}(x)(x+1)^{-1} d \lambda\right) d \mu_{\varphi}(x)
$$

for all closed loops $\Gamma: t \mapsto \lambda(t)$ in $S$. The analyticity of $\lambda \mapsto \varphi(\lambda)$ now follows from the analyticity of $w_{0,0}^{\lambda}(x)(x+1)^{-1}$ and the theorems of Cauchy and Morera. By polarization, $w_{0,0}^{\lambda}\left(H_{f}\right)\left(H_{f}+1\right)^{-1}$ is weakly analytic and hence analytic.

\section{Supplement: Background on the Fock space, etc}

Let $\mathfrak{h}$ be either $L^{2}\left(\mathbb{R}^{3}, \mathbb{C}, d^{3} k\right)$ or $L^{2}\left(\mathbb{R}^{3}, \mathbb{C}^{2}, d^{3} k\right)$. In the first case we consider $\mathfrak{h}$ as the Hilbert space of one-particle states of a scalar Boson or a phonon, and in the second case, 
of a photon. The variable $k \in \mathbb{R}^{3}$ is the wave vector or momentum of the particle. (Recall that throughout this paper, the velocity of light, $c$, and Planck's constant, $\hbar$, are set equal to 1.) The Bosonic Fock space, $\mathcal{F}$, over $\mathfrak{h}$ is defined by

$$
\mathcal{F}:=\bigoplus_{n=0}^{\infty} \mathcal{S}_{n} \mathfrak{h}^{\otimes n},
$$

where $\mathcal{S}_{n}$ is the orthogonal projection onto the subspace of totally symmetric $n$-particle wave functions contained in the $n$-fold tensor product $\mathfrak{h}^{\otimes n}$ of $\mathfrak{h}$; and $\mathcal{S}_{0} \mathfrak{h}^{\otimes 0}:=\mathbb{C}$. The vector $\Omega:=1 \bigoplus_{n=1}^{\infty} 0$ is called the vacuum vector in $\mathcal{F}$. Vectors $\Psi \in \mathcal{F}$ can be identified with sequences $\left(\psi_{n}\right)_{n=0}^{\infty}$ of $n$-particle wave functions, which are totally symmetric in their $n$ arguments, and $\psi_{0} \in \mathbb{C}$. In the first case these functions are of the form, $\psi_{n}\left(k_{1}, \ldots, k_{n}\right)$, while in the second case, of the form $\psi_{n}\left(k_{1}, \lambda_{1}, \ldots, k_{n}, \lambda_{n}\right)$, where $\lambda_{j} \in\{-1,1\}$ are the polarization variables.

In what follows we present some key definitions in the first case only limiting ourselves to remarks at the end of this appendix on how these definitions have to be modified for the second case. The scalar product of two vectors $\Psi$ and $\Phi$ is given by

$$
\langle\Psi, \Phi\rangle:=\sum_{n=0}^{\infty} \int \prod_{j=1}^{n} d^{3} k_{j} \overline{\psi_{n}\left(k_{1}, \ldots, k_{n}\right)} \varphi_{n}\left(k_{1}, \ldots, k_{n}\right) .
$$

Given a one particle dispersion relation $\omega(k)$, the energy of a configuration of $n$ noninteracting field particles with wave vectors $k_{1}, \ldots, k_{n}$ is given by $\sum_{j=1}^{n} \omega\left(k_{j}\right)$. We define the free-field Hamiltonian, $H_{f}$, giving the field dynamics, by

$$
\left(H_{f} \Psi\right)_{n}\left(k_{1}, \ldots, k_{n}\right)=\left(\sum_{j=1}^{n} \omega\left(k_{j}\right)\right) \psi_{n}\left(k_{1}, \ldots, k_{n}\right),
$$

for $n \geq 1$ and $\left(H_{f} \Psi\right)_{n}=0$ for $n=0$. Here $\Psi=\left(\psi_{n}\right)_{n=0}^{\infty}$ (to be sure that the r.h.s. makes sense we can assume that $\psi_{n}=0$, except for finitely many $n$, for which $\psi_{n}\left(k_{1}, \ldots, k_{n}\right)$ decrease rapidly at infinity). Clearly that the operator $H_{f}$ has the single eigenvalue 0 with the eigenvector $\Omega$ and the rest of the spectrum absolutely continuous.

With each function $\varphi \in \mathfrak{h}$ one associates an annihilation operator $a(\varphi)$ defined as follows. For $\Psi=\left(\psi_{n}\right)_{n=0}^{\infty} \in \mathcal{F}$ with the property that $\psi_{n}=0$, for all but finitely many $n$, the vector $a(\varphi) \Psi$ is defined by

$$
(a(\varphi) \Psi)_{n}\left(k_{1}, \ldots, k_{n}\right):=\sqrt{n+1} \int d^{3} k \overline{\varphi(k)} \psi_{n+1}\left(k, k_{1}, \ldots, k_{n}\right) .
$$


These equations define a closable operator $a(\varphi)$ whose closure is also denoted by $a(\varphi)$. Eqn (IX.4) implies the relation

$$
a(\varphi) \Omega=0 .
$$

The creation operator $a^{*}(\varphi)$ is defined to be the adjoint of $a(\varphi)$ with respect to the scalar product defined in Eq. (IX.2). Since $a(\varphi)$ is anti-linear, and $a^{*}(\varphi)$ is linear in $\varphi$, we write formally

$$
a(\varphi)=\int d^{3} k \overline{\varphi(k)} a(k), \quad a^{*}(\varphi)=\int d^{3} k \varphi(k) a^{*}(k),
$$

where $a(k)$ and $a^{*}(k)$ are unbounded, operator-valued distributions. The latter are wellknown to obey the canonical commutation relations (CCR):

$$
\left[a^{\#}(k), a^{\#}\left(k^{\prime}\right)\right]=0, \quad\left[a(k), a^{*}\left(k^{\prime}\right)\right]=\delta^{3}\left(k-k^{\prime}\right),
$$

where $a^{\#}=a$ or $a^{*}$.

Now, using this one can rewrite the quantum Hamiltonian $H_{f}$ in terms of the creation and annihilation operators, $a$ and $a^{*}$, as

$$
H_{f}=\int d^{3} k a^{*}(k) \omega(k) a(k),
$$

acting on the Fock space $\mathcal{F}$.

More generally, for any operator, $t$, on the one-particle space $\mathfrak{h}$ we define the operator $T$ on the Fock space $\mathcal{F}$ by the following formal expression $T:=\int a^{*}(k) t a(k) d k$, where the operator $t$ acts on the $k-$ variable ( $T$ is the second quantization of $t$ ). The precise meaning of the latter expression can obtained by using a basis $\left\{\phi_{j}\right\}$ in the space $\mathfrak{h}$ to rewrite it as $T:=\sum_{j} \int a^{*}\left(\phi_{j}\right) a\left(t^{*} \phi_{j}\right) d k$.

To modify the above definitions to the case of photons, one replaces the variable $k$ by the pair $(k, \lambda)$ and adds to the integrals in $k$ also the sums over $\lambda$. In particular, the creation and annihilation operators have now two variables: $a_{\lambda}^{\#}(k) \equiv a^{\#}(k, \lambda)$; they satisfy the commutation relations

$$
\left[a_{\lambda}^{\#}(k), a_{\lambda^{\prime}}^{\#}\left(k^{\prime}\right)\right]=0, \quad\left[a_{\lambda}(k), a_{\lambda^{\prime}}^{*}\left(k^{\prime}\right)\right]=\delta_{\lambda, \lambda^{\prime}} \delta^{3}\left(k-k^{\prime}\right) .
$$

One can also introduce the operator-valued transverse vector fields by

$$
a^{\#}(k):=\sum_{\lambda \in\{-1,1\}} e_{\lambda}(k) a_{\lambda}^{\#}(k),
$$

where $e_{\lambda}(k) \equiv e(k, \lambda)$ are polarization vectors, i.e. orthonormal vectors in $\mathbb{R}^{3}$ satisfying $k \cdot e_{\lambda}(k)=0$. Then in order to reinterpret the expressions in this paper for the vector 
(photon) - case one either adds the variable $\lambda$ as was mentioned above or replaces, in appropriate places, the usual product of scalar functions or scalar functions and scalar operators by the dot product of vector-functions or vector-functions and operator valued vector-functions.

\section{Acknowledgements:}

A part of this work was done while the third author was visiting ETH Zürich, ESI Vienna and IAS Princeton. He is grateful to these institutions for hospitality.

\section{References}

[1] Walid Abou Salem, J. Faupin, J. Fröhlich, I.M.Sigal. On theory of resonances in non-relativisitc QED, Preprint, 2007.

[2] V. Bach, Th. Chen, J. Fröhlich, and I. M. Sigal. Smooth Feshbach map and operatortheoretic renormalization group methods. Journal of Functional Analysis, 203, 4492, 2003.

[3] V. Bach, Th. Chen, J. Fröhlich, and I. M. Sigal. Determination of the renormalized mass for the Pauli-Fierz model. Journal of Functional Analysis, 203, 44-92, 2006.

[4] V. Bach, J. Fröhlich, and A. Pizzo. Infrared-Finite Algorithms in QED: The Groundstate of an Atom Interacting with the Quantized Radiation Field. Communications in Mathematical Physics 264, Issue: 1, 145 - 165, 2006.

[5] V. Bach, J. Fröhlich, and A. Pizzo. An infrared-finite algorithm for Rayleigh scattering amplitudes, and Bohr's frequency condition. Comm. Math. Phys. 274, no. 2, 457-486, 2007.

[6] V. Bach, J. Fröhlich, and A. Pizzo. Infrared-Finite Algorithms in QED II. The Expansion of the Groundstate of An Atom Interacting with the Quantized Radiation Field, mp_arc.

[7] V. Bach, J. Fröhlich, and I. M. Sigal. Quantum electrodynamics of confined nonrelativistic particles. Adv. in Math. , 137:299-395, 1998.

[8] V. Bach, J. Fröhlich, and I. M. Sigal. Renormalization group analysis of spectral problems in quantum field theory. Adv. in Math. , 137:205-298, 1998. 
[9] V. Bach, J. Fröhlich, and I. M. Sigal. Spectral analysis for systems of atoms and molecules coupled to the quantized radiation field. Commun. Math. Phys., 207(2):249-290, 1999.

[10] M. Berger. Nonlinearity and functional analysis. Lectures on nonlinear problems in mathematical analysis. Pure and Applied Mathematics. Academic Press, New YorkLondon, 1977.

[11] T. Chen. Operator-theoretic infrared renormalization and construction of dressed 1-particle states. Preprint mp-arc 01-310, 2001.

[12] C. Cohen-Tannoudji, J. Dupont-Roc, and G. Grynberg. Photons and Atoms - Introduction to Quantum Electrodynamics. John Wiley, New York, 1991.

[13] C. Cohen-Tannoudji, J. Dupont-Roc, and G. Grynberg. Atom-Photon Interactions Basic Processes and Applications. John Wiley, New York, 1992.

[14] J. Faupin. Resonances of the confined hydrogenoid ion and the Dicke effect in nonrelativisitc quantum electrodynamics. Preprint 2007.

[15] H. Feshbach. Unified theory of nuclear reactions. Ann. Phys., 5:357-390, 1958.

[16] J. Fröhlich, M. Griesemer and I.M. Sigal. Spectral renormalization group and limiting absorption principle for the standard model of non-relativisitc QED. Communications in Mathematical Physics, to appear, see also ArXiv, 2007

[17] J. Fröhlich, M. Griesemer and I.M. Sigal. Local decay in the standard model of non-relativisitc QED. To be posted in ArXiv

[18] M. Griesemer and D. Hasler. On the smooth Feshbach-Schur map. J. Funct. Anal., 254(9):2329-2335, 2008.

[19] M. Griesemer and D. Hasler. Analytic perturbation theory and renormalization analysis of matter coupled to quantized radiation. arXiv:0801.4458.

[20] S. Gustafson and I.M. Sigal. Mathematical Concepts of Quantum Mechanics. 2nd edition. Springer 2006.

[21] E. Hille and R.S.Phillips. Functional Analalysis and Semi-groups. AMS 1957.

[22] Tosio Kato. Perturbation theory for linear operators. Springer, 1976. 
[23] J. Schur. Über Potenzreihen die im Inneren des Einheitskreises beschränkt sind. $J$. reine u. angewandte Mathematik, 205-232, 1917.

[24] I.M. Sigal. Ground state and resonances in the standard model of the non-relativistic QED. Preprint 2007.

[25] Herbert Spohn. Dynamics of charged particles and their radiation field, Cambridge University Press, Cambridge, 2004. 